\title{
Caracterisation Hydrogeochimique Des Eaux Souterraines Des Aquiferes De Socle De La Commune De Natitingou Au Benin
}

\author{
Tinantikpa N'tcha, MSc
}

Direction Générale des Hydrocarbures et autres Combustibles Fossiles

Ministère de l'eau et des Mines, Cadjèhoun, Bénin

Wèré Gédéon Sambienou, PhD

Abdoukarim Alassane (Phd, Maître De Conférences)

Moussa Boukari (Phd, Professeur Titulaire)

Laboratoire d'Hydrologie Appliquée, Institut National de l'Eau (INE),

Université d'Abomey-Calavi,Cotonou, Bénin

Christophe Kaki (PhD, Professeur Titulaire)

Département des Sciences de la Terre, Faculté des Sciences et Techniques,

Université d'Abomey-Calavi,Abomey-Calavi, Bénin

\section{Daouda Mama (PhD, Professeur Titulaire)}

Laboratoire d'Hydrologie Appliquée, Institut National de l'Eau (INE),

Université d'Abomey-Calavi, Cotonou, Bénin

Résumé

L'objectif de cette étude est de préciser la nature hydrochimique des eaux souterraines de la commune de Natitingou et d'identifier les processus responsables de leur minéralisation, afin de contribuer à leur gestion durable. La méthodologie appliquée est basée sur une combinaison des techniques hydrochimiques et d'analyses statistiques (ACP et CHA). Les résultats issus des analyses physico-chimiques montrent une minéralisation comprise entre 40 et $3240 \mu \mathrm{S} / \mathrm{cm}$ pour une moyenne de $465,64 \mu \mathrm{S} / \mathrm{cm}$. Les eaux souterraines étudiées sont généralement acides à neutres, avec un $\mathrm{pH}$ variant entre 4,1 et 7,3. Elles se classent, de par leurs anions, en deux principaux faciès hydrogéochimiques: celui bicarbonaté $(75 \%$ des eaux) dont $45 \%$ de bicarbonaté calcique et $30 \%$ de bicarbonaté sodique et celui chloruré $(25 \%$ des eaux) dont $17 \%$ de chloruré sodique et $8 \%$ de chloruré calcique. Les différentes corrélations entre les ions majeurs et les analyses statistiques ont permis d'identifier trois processus hydrogéochimiques qui participent à la minéralisation des eaux. Le processus dominant est l'hydrolyse des minéraux 
silicatés, suivi des échanges cationiques, puis l'apport anthropogénique qui influence la minéralisation en polluant les eaux.

Mots clés : Eaux Souterraines, Faciès Hydrogéochimique, Analyse En Composantes Principales, Classification Hiérarchique Ascendante, Minéralisation, Commune De Natitingou

\title{
Hydrogeochemical Characterization of Groundwater In Basement Aquifers in the Commune of Natitingou in Benin
}

\author{
Tinantikpa N'tcha, MSc \\ Direction Générale des Hydrocarbures et autres Combustibles Fossiles \\ Ministère de l'eau et des Mines, Cadjèhoun, Bénin \\ Were Gedeon Sambienou, PhD \\ Abdoukarim Alassane (Phd, Maître De Conférences) \\ Moussa Boukari (Phd, Professeur Titulaire) \\ Laboratoire d'Hydrologie Appliquée, Institut National de l'Eau (INE), \\ Université d'Abomey-Calavi,Cotonou, Bénin \\ Christophe Kaki (PhD, Professeur Titulaire) \\ Département des Sciences de la Terre, Faculté des Sciences et Techniques, \\ Université d'Abomey-Calavi,Abomey-Calavi, Bénin \\ Daouda Mama (PhD, Professeur Titulaire) \\ Laboratoire d'Hydrologie Appliquée, Institut National de l'Eau (INE), \\ Université d'Abomey-Calavi, Cotonou, Bénin
}

\begin{abstract}
The aim of this study is to specify the hydrochemical nature of the groundwater in the commune of Natitingou and to identify the processes responsible for its mineralization, in order to contribute to its sustainable management. The methodology applied is based on a combination of hydrochemical techniques and statistical analysis (PCA and AHC). The results of the physico-chemical analyses show a mineralization between 40 and 3240 $\mu \mathrm{S} / \mathrm{cm}$ for an average of $465.64 \mu \mathrm{S} / \mathrm{cm}$. The groundwater studied is generally acidic to neutral, with a $\mathrm{pH}$ varying between 4.1 and 7.3. They are classified, by their anions, in two main hydrogeochemical facies: bicarbonate $(75 \%$ of the waters) including $45 \%$ calcium bicarbonate and $30 \%$ sodium bicarbonate
\end{abstract}


and chlorinated (25\% of the waters) including $17 \%$ sodium chloride and $8 \%$ calcium chloride. The different correlations between the major ions and the statistical analyses made it possible to identify three hydrogeochemical processes that participate in the mineralization of the waters. The dominant process is the hydrolysis of silicate minerals, followed by cationic exchanges, then the anthropogenic contribution which influences the mineralization by polluting the waters.

Keywords: Groundwater, Hydrogeochemical facies, Principal component Analysis, Ascending hierarchical classification, Mineralization, Natitingou Commune

\section{Introduction}

L'étude de l'origine et du processus de minéralisation des eaux souterraines est très importante pour l'évaluation qualitative et la gestion de la ressource surtout en régions de socle comme celle de la commune de Natitingou. Dans ces régions, la rareté et la mauvaise qualité des eaux de surface ont pour conséquence, l'exploitation intensive des eaux souterraines sans prise en compte de la vulnérabilité des aquifères à la pollution. Aussi, selon Matini et al., 2009, la composition quantitative et qualitative de l'eau souterraine en matières en suspension ou dissoutes de nature minérale ou organique, détermine sa qualité. Cette qualité peut être altérée lorsque des substances extérieures indésirables voire toxiques entrent en contact avec l'aquifère.

La commune de Natitingou est l'une des communes du Bénin où la population est majoritairement rurale. Ainsi, l'agriculture, principale activité socio-économique pourrait jouer un rôle considérable sur la qualité de l'eau. Diverses autres activités menées par les populations urbaine et rurale, ne respectent pas les normes environnementales requises. En conséquence, fournir de l'eau saine pour la consommation humaine devient de plus en plus un problème dans la Commune de Natitingou en l'occurrence, du fait de la contamination chimique des nappes. Ainsi, la prévention systématique est de loin préférable à celui qui consiste à traiter les eaux pour l'alimentation en eau potable lorsque leur qualité est détériorée. C'est dans cet esprit que s'inscrit cette étude qui a pour but d'évaluer la qualité physico-chimique des eaux souterraines de la Commune de Natitingou et d'expliquer les phénomènes à l'origine de leur minéralisation. Elle est basée sur différentes techniques hydrochimiques et d'analyse statistique multivariée.

\section{Description De La Zone D'etude}

Située au centre du département de l'Atacora dans le Nord-Ouest du Bénin, la Commune de Natitingou s'étend entre les méridiens $1^{\circ} 12^{\prime} 11^{\prime}$ 'et 
$1^{\circ} 36^{\prime} 43^{\prime \prime}$ 'de longitude est et les parallèles $10^{\circ} 00^{\prime} 00^{\prime \prime}$ ' et $10^{\circ} 27^{\prime} 36^{\prime \prime}$ ' de latitude nord (Fig. 1), avec des altitudes variant entre 300 et $450 \mathrm{~m}$. Elle s'allonge dans une sorte de vallée formée par les deux bourrelets de montagnes de quartzites qui l'entourent. Sur le plan géologique, la Commune de Natitingou, se situe dans l'unité structurale de l'Atacora (Pougnet, 1957).

La commune de Natitingou est à cheval sur trois grandes unités géomorphostructurales (Fig. 1 et 2) d'égales extensions géographiques approximativement: (i) au Nord-Ouest, la bordure orientale de l'unité structurale du Buem constituant la plaine de Tanguiéta, constituée de schistes formant des séquences de métamixites, de métasilexites, d'itabirites et de cipolins calco-dolomitiques; (ii) au Nord-Est, l'unité structurale de l'Atacora, à dominante quartzitique et subdivisée en anticlinorium de Tchakalakou, synclinorium de Toucountouna, zone monoclinale de Natitingou et anticlinorium de Kotopounga ; (iii) au Sud, la bordure occidentale des unités internes des Dahomeyides (Affaton, 1987) constituée de micaschistes quartzeux à deux micas, grenats et tourmaline, associés à des para- et orthogneiss. Ces dernières formations représentent les restes métamorphisés d'une ancienne série sédimentaire d'âge pré-panafricain, probablement kibarien (Mésoprotérozoïque), voire éburnéen selon les âges conventionnels de $1708 \pm 220$ Ma et $1925 \pm 90$ Ma obtenus sur les orthogneiss de Kouandé (Bonhomme, 1962).

\section{Cadre Hydrogeologique}

Du point de vue hydrogéologique (Fig. 2), trois différentes zones hydrogéologiques sont distinguées, correspondant aux trois unités géomorphostructurales décrites supra (Ahissou \& Goudalo, 2004) :

- la zone à réservoir essentiellement schisteux ;

- la zone à réservoir essentiellement quartzitique ;

- la zone réservoir migmatito-gneissique.

La zone à substratum schisteux : située au Nord-Ouest, elle est composée de schistes avec des intercalations de filons quartzeux ; ces schistes appartiennent à la formation de Tagayéi. C'est une zone où les venues d'eau sont liées à la présence des filons et/ou filonnets de quartz et à des intercalations de quartzites. Les épaisseurs de la couche d'altérites sont inférieures à $25 \mathrm{~m}$. Les débits sont généralement faibles et la recharge très lente (Géohydraulique, 1985).

La zone à substratum quartzitique : située au Nord-Est, elle est représentée par les sites situés sur les versants et les plateaux des collines de quartzites micacées; c'est la zone la plus vaste, avec des formations plissés à certains endroits et présentant plusieurs niveaux de fracturation, et des fractures parfois remplis de quartz. Néanmoins, sa productivité est meilleure à celle de la zone précédente (Géohydraulique, 1985). 
La zone à substratum migmatito-gneissique : située au Sud, elle est caractérisée par des couches d'altérites susceptibles d'emmagasiner une certaine quantité d'eau qui peut être localement améliorée par l'existence de différents réseaux de fractures aux mailles parfois serrées surtout si celles-ci ne sont pas remplies par les argiles d'altération. C'est la zone potentiellement favorable où on obtient un débit moyen d'exploitation atteignant $10 \mathrm{~m}^{3} / \mathrm{h}$.

Pour toutes ces zones, les nappes sont contenues dans deux types de réservoirs (Ahissou \& Goudalo, 2004) :

- un réservoir supérieur fait d'altérites et des résidus provenant de la désagrégation mécanique de la roche mère. Les forages effectués dans ces formations aquifères présentent une succession d'altérites cuirassée ou gravillonnaire, d'altérites argileuses puis d'arènes. l'épaisseur de ces altérites a été évaluée à 17 m en moyenne Sogreah-Scet (1997) ;

- un réservoir inférieur constitué de fissures et de fractures affectant le socle et favorisant l'infiltration de l'eau dans les roches cristallines. Ce type de réservoir surmonte la roche saine. Les fractures profondes s'étendent latéralement sur des dizaines de kilomètres parfois et constitue alors des réservoirs relativement favorables. 


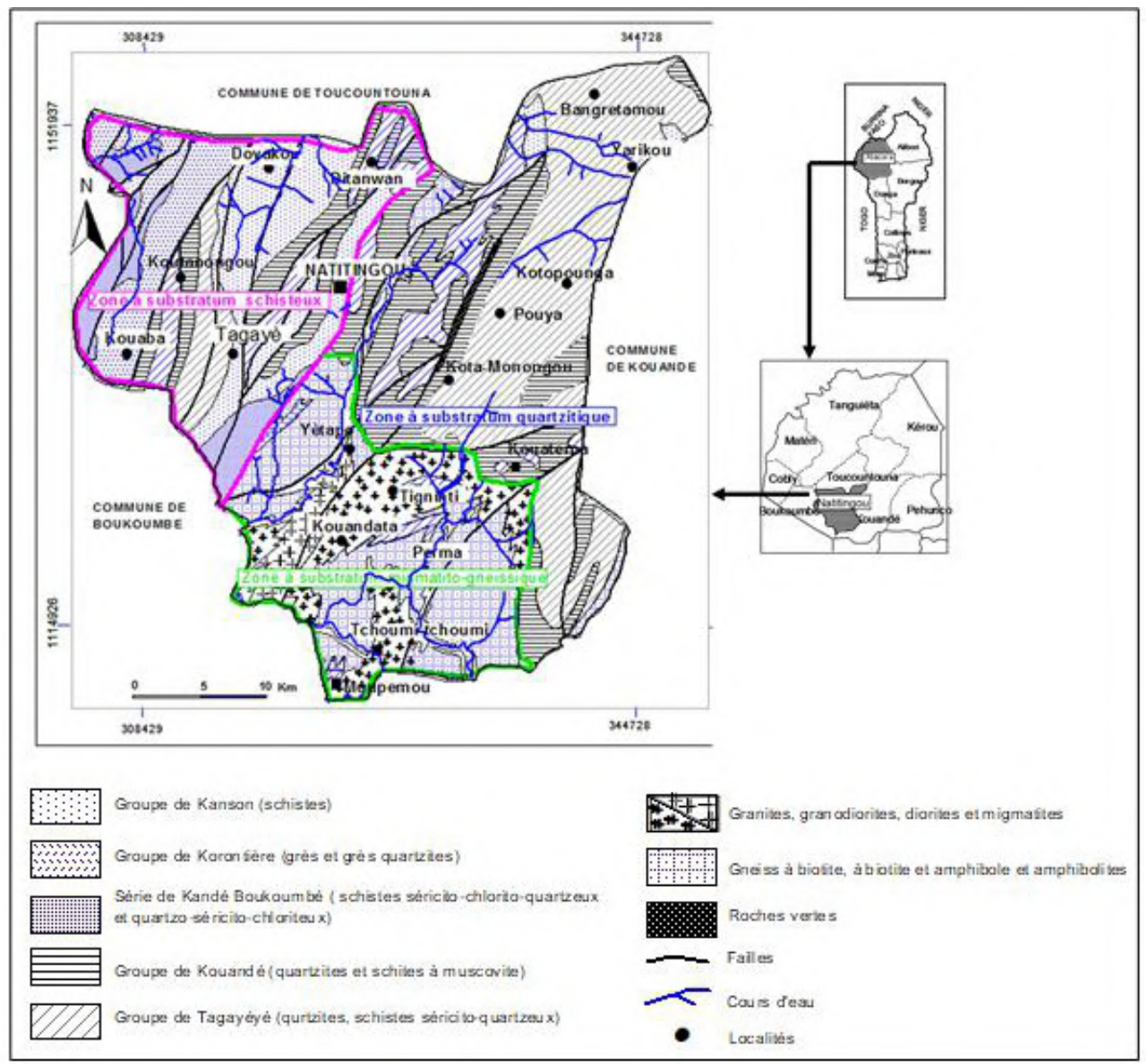

Figure 1 : Localisation et géologie de la zone d'étude

\section{Materiel Et Methodes}

\section{1- Echantillonnage}

L'échantillonnage a été réalisé sur les eaux souterraines captées par les forages et les puits dans la Commune de Natitingou (Fig. 2). Lors de cette campagne, les échantillons d'eau prélevés ont été mis dans des bouteilles en polyéthylène de capacité 1,5 litre, préalablement lavées à l'acide chlorhydrique puis à l'eau distillée. Sur le terrain, avant le remplissage des bouteilles, celles-ci ont été rincées trois fois avec l'eau à prélever. Au niveau des puits, l'eau est prélevée à l'aide d'une puisette puis embouteillée de façon à éviter l'emprisonnement des bulles d'air. Par contre, au niveau des forages, le prélèvement des échantillons d'eau a été direct à la pompe manuelle. 


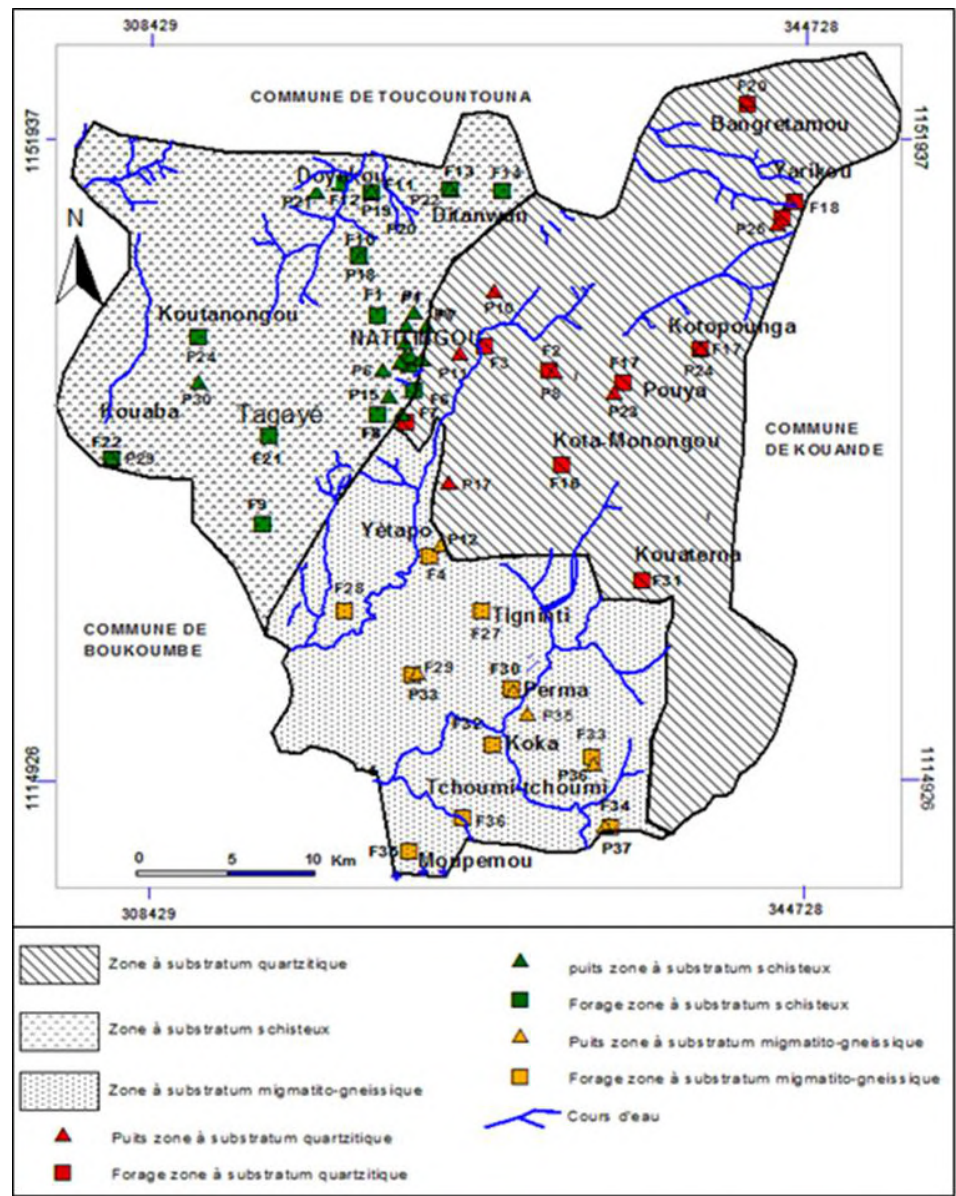

Figure 2 : Localisation des puits et forages dans la commune de Natitingou

Les échantillons ainsi prélevés ont été conservés au frais dans le laboratoire de la Société National des Eaux du Bénin (SONEB) de Natitingou pendant toute la durée de la campagne de terrain. Ils ont été ensuite transportés dans une glacière à $4{ }^{\circ} \mathrm{C}$ au laboratoire pour analyse. Sur le terrain, les mesures des paramètres physico-chimiques in-situ ont concerné la température, le $\mathrm{pH}$, la conductivité, les Solides Totaux Dissous (TDS), l'oxygène dissous. Les mesures du $\mathrm{pH}$ ont été faites avec le pH mètre RS 232 CYBER CAN 200. Le même instrument a servi aux mesures de la température. Les paramètres tels que la conductivité électrique, l'oxygène dissous et le TDS ont été mesurés à l'aide de l'appareil multiparamétrique CONSORT C532. Les mesures ont été faites courant septembre-octobre 2009.

\section{2- Analyse}

Un total de 73 échantillons d'eau prélevé, a été analysé au Laboratoire d'Hydrologie Appliquée (LHA) de l'Institut National de l'Eau de l'Université 
d'Abomey Calavi à l'aide du chromatographe ionique DIONEX-ICS 1000. Les paramètres chimiques analysés sont : $\mathrm{Na}^{+}, \mathrm{K}^{+}, \mathrm{Mg}^{2+}, \mathrm{Ca}^{2+,} \mathrm{Cl}^{-}, \mathrm{F}^{-}, \mathrm{Br}^{-}$,

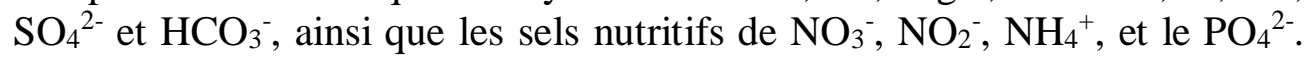
Les résultats de ces analyses sont reportés dans le tableau 1. Ces résultats ont été testés pour l'équilibre des charges ioniques selon la formule suivante (Freeze \& Cherry, 1979) :

$$
\text { BalanceIonique }=100 \times\left(\sum Z \cdot m_{c}-\sum Z \cdot m_{a}\right) /\left(\sum Z \cdot m_{c}+\sum Z \cdot m_{a}\right)
$$

$Z$ représente la valeur absolue de la valence ionique, $\mathrm{m}_{\mathrm{c}}$ la molalité des espèces cationiques et $m_{a}$ la molalité des espèces anioniques.

Pour tous les échantillons, l'erreur analytique sur l'équilibre entre les cations et les anions ne dépasse pas $5 \%(-0,7 \%$ à $5 \%)$, ce qui signifie que les résultats des analyses de laboratoire sont valables pour l'interprétation. 
Tableau 1 : Résultats des analyses physico-chimiques des eaux souterraines de la Commune de Natitingou.

\begin{tabular}{|c|c|c|c|c|c|c|c|c|c|c|c|c|c|c|c|c|c|c|}
\hline Ech. & pH & $\mathbf{T}^{\circ} \mathbf{C}$ & $\mathrm{CE}$ & $\mathrm{HCO3}$ & $\mathrm{Cl}$ & NO3 & PO4 & SO4 & $\mathrm{Na}$ & NH4 & $\mathbf{K}$ & Mg & $\mathrm{Ca}$ & NO2 & $\mathbf{F}$ & $\mathrm{Br}$ & TDS & Salinité \\
\hline FO1 & 4,86 & 29 & 43,8 & 15 & 1,4 & 0,62 & 0,06 & 0,02 & 2,4 & 0,23 & 1,2 & 0,44 & 1,91 & 0,02 & 0 & 0 & 42 & 0 \\
\hline $\mathrm{P} 1$ & 6,27 & 28 & 366 & 12 & 20 & 13,95 & 0,22 & 2,07 & 14,1 & 0,46 & 3,2 & 0,64 & 3,95 & 0 & 0 & 1,3 & 355 & 0 \\
\hline $\mathrm{P} 2$ & 4,59 & 27 & 315 & 78 & 0,3 & 0,65 & 0,18 & 0 & 23,8 & 0,36 & 3,8 & 0,73 & 3,03 & 0 & 0 & 0,1 & 304 & 0 \\
\hline P3 & 5,46 & 27 & 712 & 200 & 0,4 & 1,22 & 0,2 & 0,12 & 20 & 0,29 & 4,6 & 12,4 & 28 & 0,04 & 0 & 0 & 690 & 0 \\
\hline P4 & 5,68 & 29 & 1307 & 325 & 1,8 & 5,05 & 0,06 & 0,11 & 74 & 1,84 & 20 & 4,85 & 35,9 & 0,14 & 0 & 0,1 & 1268 & 0,4 \\
\hline P5 & 5,82 & 28 & 3240 & 12 & 285 & 437,7 & 49,4 & 0,02 & 178 & 20,2 & 73 & 13,9 & 47,5 & 0 & $\begin{array}{l}1, \\
7\end{array}$ & 0 & 71 & 1,6 \\
\hline P6 & 6,61 & 29 & 622 & 175 & 13 & 13,77 & 0,64 & 2,81 & 18,8 & 1,1 & 9,5 & 14,1 & 27,7 & 0 & 0 & 0 & 604 & 0 \\
\hline $\mathrm{P} 7$ & 5,21 & 27 & 87,6 & 2 & 21 & $\mathbf{7 0 , 3 7}$ & 0,18 & 0,05 & 4,19 & 0,07 & 1,2 & 0,37 & 2,9 & 0 & 0 & 0 & 82 & 0,1 \\
\hline $\mathrm{FO} 2$ & 4,83 & 29 & 50,7 & 15 & 2,1 & 0,35 & 0 & 0,15 & 3 & 1 & 2,2 & 0,27 & 1,6 & 0 & 0 & 0,1 & 49,1 & 0 \\
\hline P8 & 5,96 & 30 & 188 & 71 & 0,1 & 0,07 & 0,23 & 0,02 & 11,6 & 0,67 & 6,3 & 0,86 & 11,2 & 0 & 0 & 0 & 183 & 0 \\
\hline P9 & 5,96 & 29 & 1699 & 194 & 0 & 0,06 & 0,06 & 0,44 & 43,7 & 0 & 16 & 4,8 & 17,4 & 0,02 & 0 & 0 & 1648 & 0,7 \\
\hline $\mathrm{P} 10$ & 6,88 & 29 & 478 & 121 & 3,3 & 5,16 & 0,44 & 1,9 & 3,78 & 0 & 3,8 & 1,95 & 35,7 & 0 & 0 & 0 & 464 & 0 \\
\hline FO3 & 6 & 28 & 584 & 164 & 3,1 & 0,13 & 4,61 & 0,03 & 23,8 & 0,27 & 2,6 & 10,5 & 22,5 & 0 & 0 & 0 & 567 & 0 \\
\hline P11 & 6,4 & 27 & 665 & 172 & 9,4 & 4,83 & 4,77 & 0,03 & 8,64 & 0,48 & 5,1 & 10,8 & 41,3 & 0,15 & 0, & 0,1 & 645 & 0,1 \\
\hline FO4 & 5,9 & 30 & 674 & 206 & 3,1 & 1 & 1,33 & 0,03 & 18,8 & 0 & 4 & 12,4 & 28,3 & 0,97 & 0 & 0 & 653 & 0 \\
\hline $\mathrm{P} 12$ & 6 & 29 & 239 & 161 & 4,3 & 0,26 & 0,41 & 0,1 & 38,2 & 4,68 & 13 & 2,26 & 17,2 & 2,3 & 0 & 0 & 232 & 0 \\
\hline Ech. & pH & $\mathbf{T}^{\circ} \mathbf{C}$ & CE & $\mathrm{HCO3}$ & $\mathrm{Cl}$ & NO3 & PO4 & SO4 & $\mathrm{Na}$ & NH4 & $\mathbf{K}$ & Mg & $\mathrm{Ca}$ & NO2 & F & $\mathrm{Br}$ & TDS & salinité \\
\hline F5 & 4,1 & 29 & 783 & 41 & 27 & 96,28 & 0,25 & 0,31 & 24,6 & 3,07 & 14 & 4,6 & 28,9 & 0 & 0 & 0 & 760 & 0,1 \\
\hline P13 & 5,7 & 28 & 1488 & 367 & 2 & 3,3 & 1,68 & 0,11 & 61,9 & 0,81 & 33 & 10,6 & 46,2 & 0,07 & 0 & 0 & 1444 & 0,6 \\
\hline FO6 & 5,3 & 25 & 81,5 & 36 & 1,6 & 0,57 & 4,1 & 0,04 & 2,98 & 0,46 & 2 & 0,82 & 9,44 & 0,05 & 1 & 0,1 & 79 & 0 \\
\hline $\mathrm{P} 14$ & 5,1 & 29 & 373 & 35 & 14 & 50,78 & 6,71 & 0,02 & 23,4 & 0 & 2,3 & 0,7 & 9,5 & 0,08 & $\begin{array}{l}0, \\
5\end{array}$ & 0 & 361 & 0 \\
\hline FO7 & 6 & 28 & 47,8 & 5 & 1,7 & 1,17 & 1,54 & 0,04 & 0,76 & 0,08 & 1,2 & 0,16 & 1,09 & 0,02 & 0 & 0 & 46 & 0 \\
\hline P15 & 7,1 & 28 & 363 & 15 & 18 & 54,7 & 0 & 1,38 & 13,2 & 0,12 & 1,8 & 1,34 & 14,4 & 0 & 0 & 0 & 352 & 0 \\
\hline FO8 & 5,4 & 29 & 101 & 31 & 2,8 & 1,27 & 0,16 & 0,04 & 4,62 & 0 & 2,4 & 0,3 & 5,95 & 0,02 & 0 & 0 & 98 & 0 \\
\hline P16 & 6,8 & 29 & 592 & 170 & 0 & 0,21 & 1,6 & 0,08 & 5,23 & 0 & 3,2 & 1,26 & 52,6 & 0,11 & 0 & 0,3 & 573 & 0 \\
\hline FO9 & 5,3 & 28 & 69,4 & 194 & 1,9 & 0,93 & 0,13 & 0,02 & 6,62 & 1,06 & 3,2 & 1,03 & 3,8 & 0,21 & 0 & 0 & 67 & 0 \\
\hline
\end{tabular}




\begin{tabular}{|c|c|c|c|c|c|c|c|c|c|c|c|c|c|c|c|c|c|c|}
\hline P17 & 4,8 & 27 & 50,6 & 15 & 1,7 & 1,22 & 1 & 0,13 & 1,68 & 0 & 0,6 & 1 & 2,61 & 0,1 & $\begin{array}{l}0, \\
5\end{array}$ & 0 & 49 & 0 \\
\hline F10 & 5,93 & 29 & 208 & 351 & 0,1 & 0,03 & 0,47 & 0,1 & 78,1 & 0,98 & 21 & 5,14 & 37,7 & 0,02 & 0 & 0 & 202 & 0 \\
\hline P18 & 5,74 & 29 & 145 & 74 & 3 & 0,03 & 2,74 & 0,03 & 8,93 & 1,06 & 3,9 & 1,06 & 12,5 & 0,24 & 0 & 1 & 141 & 0 \\
\hline F11 & 6,39 & 30 & 416 & 150 & 1,3 & 1,23 & 1,77 & 1,22 & 16,3 & 0,4 & 2,3 & 6,62 & 16,1 & 0,12 & 0 & 0 & 404 & 0 \\
\hline P19 & 11,2 & 30 & 2120 & 280 & 0,1 & 0,02 & 0,08 & 0,03 & 3,3 & 7,1 & 29 & 0,17 & 68 & 0,14 & 0 & 0 & 400 & 0,9 \\
\hline $\mathrm{P} 20$ & 5,15 & 28 & 106 & 1 & 3,8 & 20,82 & 0,02 & 0,54 & 1,36 & 0,13 & 0,6 & 0,1 & 0,53 & 0 & 0 & 0,3 & 102 & 0 \\
\hline F12 & 6,75 & 29 & 278 & 162 & 0,2 & 0 & 0,43 & 0,03 & 22,7 & 0,4 & 1,3 & 7,27 & 29,8 & 0,01 & 0 & 0,4 & 270 & 0 \\
\hline P21 & 6,13 & 28 & 699 & 91 & 11 & 10,26 & 0,07 & 3,24 & 30,8 & 0,58 & 4,6 & 0,37 & 14,5 & 3,4 & 0 & 0 & 678 & 0,1 \\
\hline F13 & 4,97 & 30 & 44,9 & 6 & 1,3 & 0,1 & 0,37 & 0,03 & 1,34 & 0,1 & 0,8 & 0,27 & 0,92 & 0,01 & 0 & 0 & 43 & 0 \\
\hline F14 & 4,94 & 29 & 40 & 83 & 1,3 & 2,38 & 0,3 & 0,05 & 3,6 & 0,1 & 3,1 & 1,4 & 25 & 0 & 0 & 0 & 39 & 0 \\
\hline P22 & 4,82 & 28 & 271 & 15 & 11 & 37,13 & 1,4 & 1,78 & 6,93 & 0,47 & 8,1 & 2,55 & 9,57 & 0 & 0 & 0 & 263 & 0 \\
\hline F15 & 4,93 & 28 & 137 & 142 & 8,2 & 10,31 & 0,4 & 0,23 & 37,8 & 4,4 & 21 & 0,93 & 10,5 & 0 & 0 & 0 & 133 & 0 \\
\hline F16 & 4,76 & 28 & 44,6 & 3 & 2,9 & 4,32 & 0,23 & 0,06 & 1,91 & 0,12 & 0,9 & 0,35 & 1,38 & 0 & 0 & 0,1 & 43 & 0 \\
\hline F17 & 4,71 & 28 & 145 & 41 & 0,2 & 0,55 & 0,13 & 0,06 & 7,9 & 0,3 & 2,7 & 0,88 & 3,71 & 0,03 & 0 & 0 & 141 & 0 \\
\hline P23 & 5,93 & 27 & 263 & 56 & 9,7 & 27,11 & 3,43 & 0,02 & 15,5 & 0,92 & 8,7 & 1,08 & 13,2 & 0,7 & $\begin{array}{l}0, \\
4\end{array}$ & 0 & 255 & 0 \\
\hline F18 & 4,92 & 27 & 40,1 & 3 & 0,1 & 0,01 & 0,47 & 0,04 & 1,98 & 0,11 & 0,5 & 0,16 & 1,9 & 0,02 & 0 & 0 & 39 & 0 \\
\hline P24 & 4,53 & 26 & 604 & 3 & 26 & 133,7 & 0,62 & 0,73 & 19 & 0,34 & 11 & 3,3 & 14 & 0 & 0 & 0 & 586 & 0 \\
\hline F19 & 4,69 & 28 & 122 & 37 & 5,5 & 18,34 & 0,77 & 0,3 & 4,68 & 0,13 & 2,5 & 0,51 & 3,03 & 0 & 0 & 0 & 118 & 0 \\
\hline P25 & 5,52 & 28 & 259 & 54 & 5,6 & 18,05 & 0,33 & 0,08 & 17,7 & 0,52 & 4,3 & 0,35 & 5,8 & 0 & 0 & 0 & 251 & 0 \\
\hline F20 & 4,6 & 28 & 444 & 4 & 18 & 25,62 & 0,46 & 1,67 & 22,6 & 0,25 & 14 & 1,56 & 6,25 & 0,3 & 0 & 0 & 431 & 0 \\
\hline P26 & 6,21 & 27 & 572 & 80 & 17 & 39,43 & 7,67 & 0,02 & 16,1 & 0 & 12 & 1,35 & 29,2 & 0 & 0 & 0 & 556 & 0 \\
\hline F21 & 4,6 & 28 & 90,1 & 35 & 6,1 & 10,51 & 0,05 & 0,36 & 3,73 & 0,11 & 1,7 & 0,43 & 2,92 & 0 & 0 & 0 & 87 & 0 \\
\hline P28 & 5,59 & 27 & 636 & 31 & 31 & 97,78 & 0,4 & 5,68 & 17,7 & 0,63 & 41 & 3,48 & 14,1 & 0 & 0 & 0 & 617 & 0,1 \\
\hline F22 & 5,12 & 29 & 162 & 42 & 6,2 & 14,32 & 11 & 0,04 & 2,47 & 0 & 3,6 & 1,41 & 11,4 & 0,4 & 0 & 0 & 157 & 0 \\
\hline F23 & 5,84 & 29 & 177 & 22 & 3,4 & 6,03 & 1,52 & 0,03 & 1,56 & 0 & 3,2 & 1,35 & 11,5 & 0,3 & 0 & 0 & 172 & 0 \\
\hline P29 & 5,64 & 30 & 106 & 67 & 4,1 & 0,13 & 0,13 & 0,2 & 17,1 & 0,9 & 6 & 1,25 & 7,83 & 0,08 & 0 & 0 & 103 & 0 \\
\hline F24 & 5,1 & 29 & 75,8 & 18 & 2,4 & 4,2 & 0,22 & 0,17 & 3,76 & 0,15 & 2,9 & 0,85 & 3,44 & 0 & 0 & 0 & 72 & 0 \\
\hline Ech. & pH & $\mathbf{T}^{\circ} \mathbf{C}$ & CE & $\mathrm{HCO3}$ & Cl & NO3 & PO4 & SO4 & $\mathrm{Na}$ & NH4 & $\mathbf{K}$ & Mg & Ca & $\mathrm{NO2}$ & $\mathbf{F}$ & $\mathrm{Br}$ & TDS & salinité \\
\hline P30 & 6,24 & 27 & 174 & 23 & 2,7 & 2,46 & 0 & 0,63 & 2,33 & 0,2 & 0,9 & 0,96 & 5,26 & 0,02 & 0 & 0 & 169 & 0 \\
\hline F25 & 4,81 & 29 & 57,8 & 47 & 2,8 & 0,08 & 0,1 & 1,66 & 4,96 & 0,11 & 1,4 & 0,13 & 2,33 & 0 & 0 & 0 & 56 & 0 \\
\hline
\end{tabular}




\begin{tabular}{|c|c|c|c|c|c|c|c|c|c|c|c|c|c|c|c|c|c|c|}
\hline F26 & 5,62 & 29 & 148 & 86 & 1,2 & 1,06 & 0,11 & 1,45 & 4,68 & 0,2 & 1,7 & 3,37 & 6,5 & 0 & 0 & 0 & 144 & 0 \\
\hline P31 & 5,74 & 28 & 93,3 & 36 & 0,1 & 0,3 & 0,4 & 0,04 & 8,16 & 0,2 & 2,1 & 0,74 & 2,05 & 0 & 0 & 0 & 90 & 0 \\
\hline F27 & 6,47 & 29 & 473 & 114 & 11 & 9,61 & 0,83 & 5,64 & 7,83 & 0,13 & 7,7 & 1,28 & 26,6 & 0 & 0 & 0 & 460 & 0 \\
\hline P32 & 6,35 & 28 & 485 & 45 & 11 & 11,83 & 0,75 & 7,17 & 16,8 & 1,64 & 13 & 0,67 & 5,65 & 0 & 0 & 0 & 470 & 0 \\
\hline F28 & 5,6 & 30 & 375 & 92 & 1,4 & 0,05 & 1,11 & 0,3 & 17,8 & 0 & 5,3 & 3,05 & 14,3 & 0,04 & 0 & 0 & 364 & 0 \\
\hline F29 & 6,25 & 29 & 182 & 35 & 4,4 & 1,11 & 0,44 & 1,04 & 17,7 & 0,31 & 3,6 & 1,6 & 15,5 & 0 & 0 & 0 & 176 & 0 \\
\hline F30 & 6,26 & 30 & 403 & 285 & 1,3 & 0 & 1,12 & 0,4 & 5,3 & 0,09 & 1,2 & 2,01 & 2,85 & 0 & 0 & 0 & 391 & 0 \\
\hline P33 & 6,32 & 29 & 442 & 45 & 27 & 30,43 & 0,17 & 2,12 & 25,9 & 0,5 & 2,6 & 0,32 & 14,3 & 0 & 0 & 0 & 428 & 0 \\
\hline F31 & 6,4 & 29 & 888 & 39 & 0,1 & 0,06 & 0,26 & 0,17 & 31,2 & 0,47 & 5,1 & 10,1 & 52 & 0 & 0 & 0,1 & 861 & 0,2 \\
\hline P34 & 5,9 & 28 & 205 & 56 & 5,1 & 0,1 & 3,4 & 0,12 & 5,81 & 0,22 & 2,1 & 0,52 & 12,5 & 0,01 & 0 & 0 & 199 & 0 \\
\hline F32 & 5,52 & 28 & 132 & 236 & 2,1 & 0,58 & 0,35 & 0,19 & 3 & 0,14 & 1,1 & 3,2 & 4,35 & 0 & 0 & 0,1 & 128 & 0 \\
\hline F33 & 6,6 & 29 & 938 & 236 & 12 & 9,9 & 5,63 & 0,02 & 24,3 & 0,8 & 6,1 & 21,2 & 37,8 & 0 & 4, & 0 & 910 & 0,2 \\
\hline P35 & 6,62 & 28 & 858 & 201 & 14 & 9,26 & 1,86 & 5,43 & 23,7 & 0,4 & 5,3 & 5,02 & 45,1 & 0 & 0 & 0,6 & 831 & 0,2 \\
\hline F34 & 7,3 & 31 & 1661 & 102 & 5,4 & 0,2 & 0,04 & 175 & 55,3 & 2,57 & 22 & 19,8 & 58,3 & 0 & 0 & 0 & 1610 & 0,7 \\
\hline P36 & 5,92 & 30 & 332 & 36 & 24 & 18,8 & 0 & 5,38 & 16 & 0,38 & 4,3 & 3,54 & 9,01 & 0 & 0 & 0,8 & 322 & 0 \\
\hline F35 & 6,33 & 30 & 551 & 315 & 1,9 & 0,24 & 0,08 & 0,98 & 14 & 0,41 & 7,3 & 0,7 & 14,3 & 0 & 0 & 0 & 534 & 0 \\
\hline P37 & 5,44 & 31 & 122 & 132 & 2,2 & 11,72 & 0,16 & 3,24 & 32,8 & 1,5 & 12 & 3,07 & 16,1 & 0 & 0 & 0 & 118 & 0 \\
\hline F36 & 6,4 & 30 & 1201 & 95 & 4 & 0,02 & 228 & 0,62 & 27,6 & 0 & 6,2 & 14,4 & 70,3 & 0 & $\begin{array}{l}1, \\
5\end{array}$ & 0 & 1165 & 0,4 \\
\hline F37 & 6,7 & 30 & 845 & 224 & 0 & 0,18 & 0,08 & 0,13 & 6,48 & 0 & 2 & 5,52 & 14 & 0 & 0 & 0,1 & 820 & 0,2 \\
\hline
\end{tabular}




\section{3- Traitement des données}

Le traitement des données collectées sur les eaux souterraines de la Commune de Natitingou a été réalisé à l'aide des méthodes hydrochimiques et statistique mutlivariée. Pour l'étude hydrochimique, le diagramme de Piper a été nécessaire pour la classification hydrochimique des eaux. En effet, l'utilisation de ce diagramme dans le domaine de l'hydrochimie par bon nombre d'auteurs (Biémi, 1992 ; Yaméogo, 2008 ; Abdou Babaye, 2012 ; Sambiénou et $a l ., 2018 \mathrm{~b}$ ) a conduit à de très bons résultats. L'approche statistique qui a été utilisée pour étudier les phénomènes à l'origine de la minéralisation des eaux, le regroupement des eaux et identifier les facteurs responsables de ces regroupements est basée sur l'Analyse en Composantes Principales (ACP) et la Classification Hiérarchique Ascendante (CHA). Leur application dans l'étude hydrochimique des eaux a été réalisée par plusieurs auteurs en Afrique et dans le monde avec des résultats très significatifs (Biémi, 1992 ; Yaméogo, 2008 ; Sambiénou et al., 2018 b). L'applicabilité de la technique exploratoire ACP repose sur le choix du nombre d'axes représentant le maximum d'inertie conservée avec le minimum possible de facteurs. En général, l'ACP d'une région n'est valable que lorsque les plans factoriels étudiés retiennent au moins 70\% d'informations (Faillat \& Drogue, 1993 ; Abdou Babaye, 2012; Sambiénou, 2019). En dessous de cette limite, on considère que l'étude de la région n'a pas tenu compte d'une grande quantité d'informations. Les analyses statistiques ont porté sur les 18 variables suivants : $\mathrm{pH}, \mathrm{T}^{\circ} \mathrm{C}, \mathrm{TDS}, \mathrm{CE}, \mathrm{Na}^{+}, \mathrm{K}^{+}, \mathrm{Mg}^{2+}, \mathrm{Ca}^{2+}, \mathrm{Cl}^{-}, \mathrm{F}^{-}, \mathrm{Br}^{-}, \mathrm{SO}_{4}{ }^{2-}, \mathrm{HCO}_{3}^{-}$, $\mathrm{NO}_{3}{ }^{2-}, \mathrm{NO}_{2}{ }^{-}, \mathrm{NH}_{4}{ }^{+}, \mathrm{PO}_{4}{ }^{2-}$ et la Salinité.

Les analyses statistiques ont été réalisées à l'aide du logiciel XLSTAT 2015. Les valeurs propres, les poids factoriels des variables et les poids factoriels des individus issus de ce traitement sont reportés dans les tableaux 2,3 et 4 ci-après :

Tableau 2: Valeurs propres de l'ACP

\begin{tabular}{cccccc}
\hline & $\mathrm{F} 1$ & $\mathrm{~F} 2$ & $\mathrm{~F} 3$ & $\mathrm{~F} 4$ & $\mathrm{~F} 5$ \\
\hline $\begin{array}{c}\text { Valeur } \\
\text { propre }\end{array}$ & 6,767 & 3,244 & 1,365 & 1,209 & 1,046 \\
$\begin{array}{c}\text { Variabilité } \\
(\%)\end{array}$ & 37,596 & 18,022 & 7,582 & 6,719 & 5,809 \\
$\%$ cumulé & 37,596 & 55,619 & 63,200 & 69,919 & 75,727 \\
\hline
\end{tabular}


Tableau 3 : Poids factoriels des variables

\begin{tabular}{cccccc}
\hline & $\mathrm{F} 1$ & $\mathrm{~F} 2$ & $\mathrm{~F} 3$ & $\mathrm{~F} 4$ & $\mathrm{~F} 5$ \\
\hline $\mathrm{pH}$ & 0,415 & $\mathbf{0 , 5 6 8}$ & $-0,299$ & 0,404 & 0,173 \\
$\mathrm{~T}^{\circ} \mathrm{C}$ & 0,191 & 0,471 & $-0,201$ & $-0,010$ & $-0,058$ \\
$\mathrm{CE}$ & $\mathbf{0 , 9 4 6}$ & 0,094 & $-0,095$ & $-0,010$ & 0,037 \\
$\mathrm{HCO} 3$ & 0,415 & $\mathbf{0 , 7 5 2}$ & $-0,024$ & 0,314 & 0,013 \\
$\mathrm{Cl}$ & $\mathbf{0 , 7 2 5}$ & $\mathbf{- 0 , 6 0 8}$ & 0,007 & 0,151 & $-0,060$ \\
$\mathrm{NO} 3$ & $\mathbf{0 , 6 6 5}$ & $\mathbf{- 0 , 6 6 0}$ & 0,029 & 0,067 & $-0,079$ \\
$\mathrm{PO} 4$ & 0,361 & 0,157 & $\mathbf{0 , 6 1 9}$ & 0,102 & 0,141 \\
$\mathrm{SO} 4$ & 0,298 & 0,389 & $-0,312$ & $-0,455$ & $-0,362$ \\
$\mathrm{Na}$ & $\mathbf{0 , 8 3 6}$ & $-0,292$ & $-0,078$ & $-0,145$ & 0,014 \\
$\mathrm{NH} 4$ & $\mathbf{0 , 7 8 9}$ & $-0,472$ & $-0,174$ & 0,146 & 0,059 \\
$\mathrm{~K}$ & $\mathbf{0 , 8 3 4}$ & $-0,331$ & $-0,191$ & $-0,091$ & $-0,017$ \\
$\mathrm{Mg}$ & $\mathbf{0 , 6 6 6}$ & 0,408 & 0,325 & $-0,220$ & $-0,112$ \\
$\mathrm{Ca}$ & $\mathbf{0 , 7 3 2}$ & 0,499 & 0,056 & 0,066 & 0,083 \\
$\mathrm{NO} 2$ & 0,001 & 0,004 & $-0,246$ & $-0,129$ & $\mathbf{0 , 8 6 9}$ \\
$\mathrm{F}$ & 0,436 & 0,018 & $\mathbf{0 , 6 8 4}$ & 0,110 & 0,073 \\
$\mathrm{Br}$ & $-0,051$ & 0,126 & $-0,181$ & $\mathbf{0 , 6 6 2}$ & $-0,260$ \\
$\mathrm{TDS}$ & $\mathbf{0 , 5 1 3}$ & $\mathbf{0 , 5 8 9}$ & 0,021 & $-0,332$ & 0,003 \\
Salinité & $\mathbf{0 , 9 1 5}$ & $-0,074$ & $-0,116$ & $-0,006$ & $-0,027$ \\
\hline
\end{tabular}

Tableau 4 : Poids factoriels des individus

\begin{tabular}{llllll}
\hline & F1 & F2 & F3 & F4 & F5 \\
\hline FO1 & 0,719 & 0,141 & 0,007 & 0,015 & 0,013 \\
P1 & 0,027 & 0,000 & 0,035 & 0,419 & 0,073 \\
P2 & 0,371 & 0,260 & 0,019 & 0,060 & 0,020 \\
P3 & 0,004 & 0,013 & 0,093 & 0,178 & 0,003 \\
P4 & 0,438 & 0,018 & 0,026 & 0,087 & 0,001 \\
P5 & 0,749 & 0,237 & 0,000 & 0,005 & 0,000 \\
P6 & 0,168 & 0,265 & 0,007 & 0,013 & 0,006 \\
P7 & 0,245 & 0,422 & 0,018 & 0,000 & 0,012 \\
FO2 & 0,596 & 0,135 & 0,000 & 0,005 & 0,026 \\
P8 & 0,381 & 0,014 & 0,033 & 0,000 & 0,006 \\
P9 & 0,283 & 0,054 & 0,016 & 0,094 & 0,000 \\
P10 & 0,008 & 0,403 & 0,032 & 0,039 & 0,004 \\
FO3 & 0,017 & 0,282 & 0,072 & 0,027 & 0,003 \\
P11 & 0,084 & 0,236 & 0,034 & 0,006 & 0,005 \\
FO4 & 0,048 & 0,394 & 0,001 & 0,011 & 0,145 \\
P12 & 0,002 & 0,016 & 0,106 & 0,007 & 0,663 \\
FO5 & 0,135 & 0,210 & 0,003 & 0,163 & 0,027 \\
P13 & 0,478 & 0,023 & 0,002 & 0,119 & 0,000
\end{tabular}




\begin{tabular}{|c|c|c|c|c|c|}
\hline FO6 & 0,176 & 0,140 & 0,182 & 0,004 & 0,001 \\
\hline P14 & 0,115 & 0,186 & 0,120 & 0,042 & 0,003 \\
\hline F7 & 0,646 & 0,112 & 0,000 & 0,006 & 0,001 \\
\hline P15 & 0,053 & 0,036 & 0,010 & 0,028 & 0,001 \\
\hline FO8 & 0,795 & 0,083 & 0,000 & 0,002 & 0,007 \\
\hline P16 & 0,005 & 0,435 & 0,026 & 0,222 & 0,003 \\
\hline FO9 & 0,681 & 0,186 & 0,000 & 0,009 & 0,007 \\
\hline P17 & 0,497 & 0,180 & 0,123 & 0,005 & 0,000 \\
\hline F10 & 0,104 & 0,000 & 0,018 & 0,008 & 0,000 \\
\hline P18 & 0,073 & 0,001 & 0,043 & 0,406 & 0,036 \\
\hline F11 & 0,005 & 0,487 & 0,009 & 0,012 & 0,001 \\
\hline P19 & 0,341 & 0,125 & 0,116 & 0,126 & 0,029 \\
\hline P20 & 0,614 & 0,143 & 0,001 & 0,046 & 0,063 \\
\hline $\mathrm{F} 12$ & 0,000 & 0,389 & 0,013 & 0,323 & 0,020 \\
\hline P21 & 0,000 & 0,000 & 0,069 & 0,030 & 0,740 \\
\hline F13 & 0,560 & 0,063 & 0,000 & 0,008 & 0,020 \\
\hline F14 & 0,494 & 0,073 & 0,018 & 0,021 & 0,010 \\
\hline P22 & 0,329 & 0,344 & 0,026 & 0,109 & 0,058 \\
\hline F15 & 0,002 & 0,487 & 0,016 & 0,035 & 0,005 \\
\hline \multirow[t]{2}{*}{ F16 } & 0,700 & 0,190 & 0,010 & 0,010 & 0,024 \\
\hline & $\mathrm{F} 1$ & $\mathrm{~F} 2$ & F3 & $\mathrm{F} 4$ & F5 \\
\hline F17 & 0,618 & 0,204 & 0,024 & 0,041 & 0,010 \\
\hline P23 & 0,085 & 0,212 & 0,009 & 0,005 & 0,331 \\
\hline F17 & 0,593 & 0,222 & 0,024 & 0,015 & 0,004 \\
\hline P24 & 0,001 & 0,361 & 0,037 & 0,060 & 0,015 \\
\hline F18 & 0,568 & 0,292 & 0,027 & 0,034 & 0,017 \\
\hline P25 & 0,458 & 0,277 & 0,002 & 0,011 & 0,005 \\
\hline F19 & 0,088 & 0,445 & 0,000 & 0,256 & 0,003 \\
\hline P26 & 0,004 & 0,016 & 0,007 & 0,000 & 0,003 \\
\hline F20 & 0,636 & 0,217 & 0,020 & 0,028 & 0,022 \\
\hline P28 & 0,153 & 0,181 & 0,011 & 0,039 & 0,013 \\
\hline F21 & 0,452 & 0,030 & 0,002 & 0,026 & 0,051 \\
\hline F22 & 0,525 & 0,006 & 0,022 & 0,001 & 0,046 \\
\hline P29 & 0,364 & 0,043 & 0,027 & 0,004 & 0,001 \\
\hline $\mathrm{F} 23$ & 0,627 & 0,087 & 0,000 & 0,015 & 0,020 \\
\hline P30 & 0,490 & 0,010 & 0,000 & 0,029 & 0,000 \\
\hline F24 & 0,681 & 0,106 & 0,003 & 0,017 & 0,023 \\
\hline F25 & 0,528 & 0,007 & 0,000 & 0,001 & 0,023 \\
\hline P31 & 0,717 & 0,118 & 0,000 & 0,001 & 0,004 \\
\hline
\end{tabular}




\begin{tabular}{llllll} 
F26 & 0,002 & 0,327 & 0,023 & 0,077 & 0,000 \\
P32 & 0,018 & 0,048 & 0,064 & 0,000 & 0,002 \\
F27 & 0,106 & 0,150 & 0,012 & 0,011 & 0,007 \\
F28 & 0,429 & 0,001 & 0,031 & 0,017 & 0,002 \\
F29 & 0,155 & 0,172 & 0,028 & 0,011 & 0,003 \\
P33 & 0,023 & 0,015 & 0,073 & 0,001 & 0,004 \\
F30 & 0,255 & 0,501 & 0,000 & 0,003 & 0,000 \\
P34 & 0,686 & 0,011 & 0,001 & 0,014 & 0,000 \\
F31 & 0,768 & 0,006 & 0,007 & 0,014 & 0,034 \\
F32 & 0,184 & 0,118 & 0,333 & 0,002 & 0,001 \\
P35 & 0,121 & 0,284 & 0,018 & 0,247 & 0,020 \\
F33 & 0,330 & 0,269 & 0,069 & 0,145 & 0,076 \\
P36 & 0,005 & 0,062 & 0,051 & 0,400 & 0,106 \\
F34 & 0,015 & 0,263 & 0,100 & 0,017 & 0,002 \\
P37 & 0,024 & 0,029 & 0,029 & 0,039 & 0,019 \\
F35 & 0,212 & 0,182 & 0,357 & 0,004 & 0,016 \\
F36 & 0,033 & 0,542 & 0,020 & 0,020 & 0,001 \\
\hline
\end{tabular}

L'application des différentes méthodes retenues a permis de décrire la minéralisation des eaux des aquifères du socle de la commune de Natitingou ainsi que les mécanismes mis en jeu.

\section{Resultats Et Discussion}

\section{1- Paramètres Physico-Chimiques Des Eaux}

Les résultats du tableau 1 et de la figure 3 montrent que les valeurs de TDS des eaux étudiées sont très variables d'une zone hydrogéologique à l'autre comme le montre, avec $89 \%$ de ces valeurs comprises entre 0 et 800 $\mathrm{mg} / \mathrm{L}$ et $11 \%$ entre 800 et $1500 \mathrm{mg} / \mathrm{L}$. On note que les valeurs élevées concernent les eaux du substratum migmatito-gneissique et celles de la zone schisteuse, la zone quartzitique affichant les valeurs les plus faibles. 


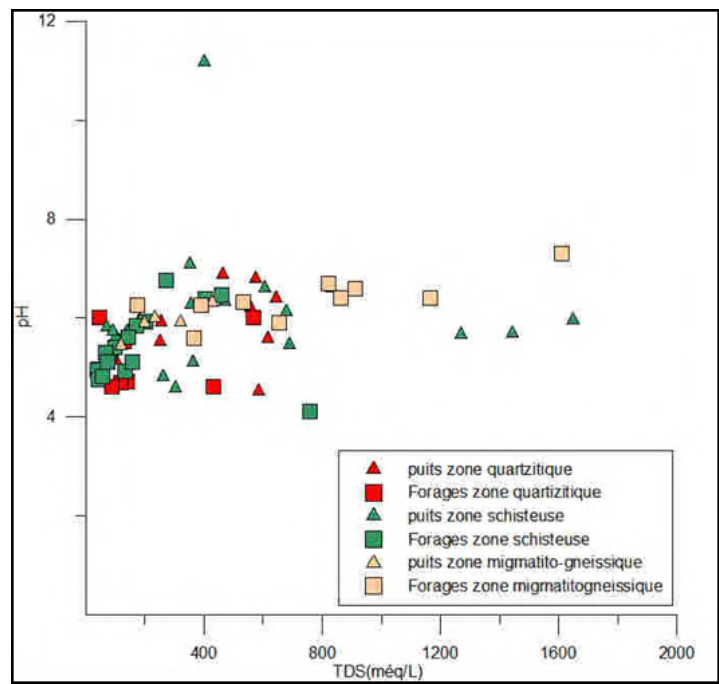

Figure 3: Relation $\mathrm{pH} / \mathrm{TDS}$ dans les eaux étudiées

Les figures 3 et 4 montrent que les eaux de la zone à substratum migmatito-gneissique ont un $\mathrm{pH}$ compris entre 5,3 et 7,3. Par contre, les eaux des forages situés dans la zone schisteuse et celle quartzitique plus acides, ont des $\mathrm{pH}$ situés entre 4,1 et 6,75 . Les eaux de la zone quartzitique présentent les $\mathrm{pH}$ les plus faibles compris entre 4,6 et 5,12. Ce caractère acide des eaux souterraines de la commune pourrait s'expliquer par une forte production du $\mathrm{CO}_{2}$ biogénique dans le milieu (Faillat \& Drogue, 1993). La commune de Natitingou étant une zone dont les principales activités rémunératrices sont l'agriculture et l'élevage, l'activité biologique peut libérer de fortes quantités de $\mathrm{CO}_{2}$ dans le sol qui en solution dans l'eau donne de l'acide carbonique selon la réaction (1) :

$$
\mathrm{CO}_{2}+\mathrm{H}_{2} \mathrm{O} \rightleftarrows \mathrm{H}_{2} \mathrm{CO}_{3}(\mathbf{1})
$$


Les variations des valeurs de $\mathrm{pH}$ sont en relation avec la pression partielle du $\mathrm{CO}_{2}$ dans l'eau, et plus cette pression augmente, plus le $\mathrm{pH}$ diminue (Figure 4) et tend à se stabiliser à 4,5.

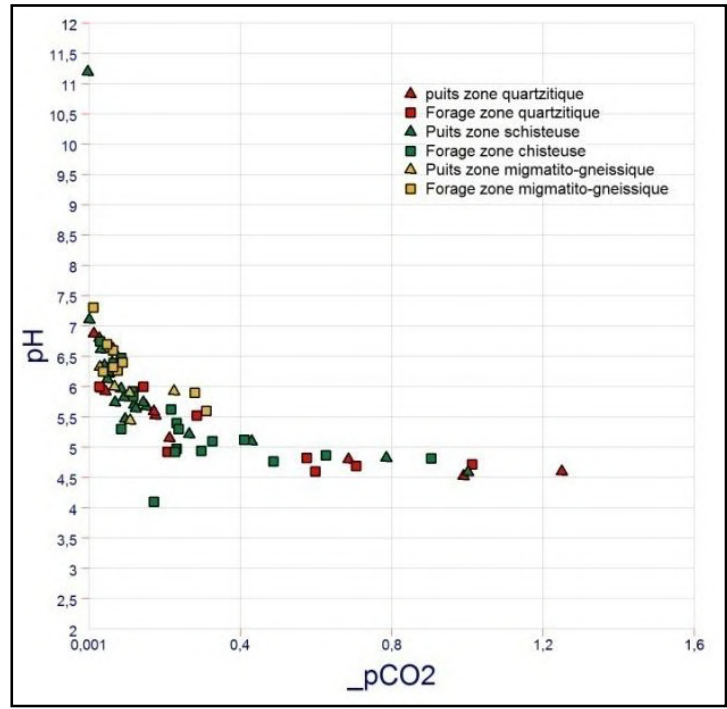

Figure 4: Relation $\mathrm{pH} / \mathrm{pCO}_{2}$ dans les eaux étudiées

La conductivité électrique (CE) des eaux de la plaine à substratum métamorphique ont des conductivités variables qui vont de moins de 100 $\mu \mathrm{S} / \mathrm{cm}(69,4 \mu \mathrm{S} / \mathrm{cm}$ au niveau du forage $\mathrm{A} 3040$ de Koussantikou) à plus de $1500 \mu \mathrm{S} / \mathrm{cm}(1661 \mu \mathrm{S} / \mathrm{cm}$ au niveau du forage A2925 de Pam-Pam). La minéralisation des eaux de cette zone est donc très variable allant des eaux très peu minéralisées aux eaux fortement minéralisées. Les eaux de la zone quartzitique présentent des conductivités variant entre 40 et $444 \mu \mathrm{S} / \mathrm{cm}$ tandis que celles de la zone schisteuse présentent des conductivités comprises entre 47,8 et $783 \mu \mathrm{S} / \mathrm{cm}$. Ces deux dernières zones présentent donc des eaux faiblement minéralisées. Les conductivités électriques de ces deux dernières zones, montrent que certains points d'eau sont très faiblement minéralisés et d'autres présentent les minéralisations importantes. 


\section{2- Paramètres chimiques des eaux}

L'essentiel de nos échantillons, reportés sur le diagramme Piper, correspondent à des faciès bicarbonatés calciques et bicarbonatés sodiques. Le reste est représenté par les faciès chlorurés sodiques et chlorurés calciques (Figure 5). Il s'agit des eaux bicarbonatées à $75 \%$ dont $45 \%$ de bicarbonatés calciques et $30 \%$ de bicarbonatés sodique et à $25 \%$ des eaux chlorurées dont $16,5 \%$ chlorurés calciques et $8.5 \%$ de chlorurés sodiques.

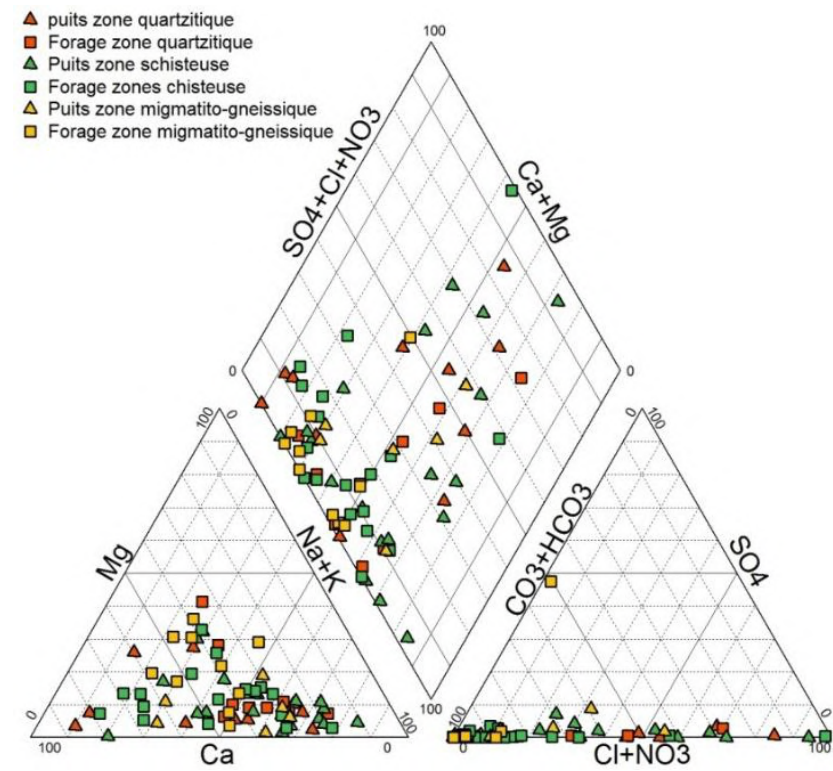

Figure 5: Diagramme de Piper des eaux de puits et de forages (septembre 2009)

L'utilisation de $\mathrm{HCO}_{3}{ }^{-}$comme indicateur du processus chimique dominant de production d'ions dans les nappes de socle, est à relier avec le mode de sa formation. Les bicarbonates sont produits suite à l'apport du $\mathrm{CO}_{2}$ par l'infiltration qui, par réaction d'hydrolyse, permet d'une part la formation du $\mathrm{HCO}_{3}{ }^{-}$et d'autre part l'augmentation du $\mathrm{pH}$ en supposant une évolution en milieu fermé (Abdou Babaye, 2012). C'est partant de ces faits que les hydrogéologues utilisent le $\mathrm{HCO}_{3}{ }^{-}$et le $\mathrm{pH}$ pour mettre en relation le contact eau-roche et la production d'ions dans l'eau.

Le tableau 5, avec les coefficients de corrélation, met en évidence les relations existantes entre les teneurs en cations majeurs pris individuellement et les bicarbonates. Les différentes valeurs montrent qu'il existe d'une part, une corrélation moyenne entre les ions bicarbonates et les ions $\mathrm{Mg}^{2+}(\mathrm{r}=0,53)$ et d'autre part, entre les ions bicarbonates et les ions $\mathrm{Ca}^{2+}(\mathrm{r}=0,66)$. Par contre il n'est noté aucune corrélation entre les ions bicarbonates et les ions $\mathrm{Na}^{+}$et $\mathrm{K}^{+}$. La dépendance moyenne entre les ions bicarbonates et les ions $\mathrm{Mg}^{2+}, \mathrm{Ca}^{2+}$ d'une part, et la non corrélation des ions $\mathrm{Na}^{+}$et $\mathrm{K}^{+}$avec $\mathrm{HCO}_{3}{ }^{-}$d'autre part, montrent qu'en dehors de l'hydrolyse il existerait un autre mécanisme de mise 
en solution de ces ions. Par ailleurs, les teneurs en $\mathrm{Na}^{+}$excèdent celles de $\mathrm{Cl}^{-}$ (Figure 6a). Cet excès peut être expliqué par les phénomènes d'échange cationique qui s'opèrent via les minéraux argileux (Abid, 2000). Ce phénomène est confirmé par la libération des ions $\mathrm{Na}^{+}$et la fixation des ions $\mathrm{Ca}^{2+}$ (Figure 7). Cette situation est également notée dans le rapport $\mathrm{K}^{+} / \mathrm{Cl}^{-}$ (Figure 6b). La figure 6c illustrant la relation $\mathrm{Ca}^{2+} / \mathrm{SO}_{4}{ }^{2-}$, montre un excès des ions $\mathrm{Ca}^{2+}$ qui pourrait provenir soit de dissolution du gypse ou de l'anhydrite soit des échanges de base avec les minéraux présents en abondance dans les niveaux aquifères (Abid, 2000). En effet, à l'image de toutes les zones chaudes, la zone d'étude est probablement soumise à une évaporation importante due aux températures élevées pendant les saisons sèches. Ce phénomène favoriserait la formation et la concentration de certains sels minéraux comme le gypse ( $\left.\mathrm{CaSO} 4,2 \mathrm{H}_{2} \mathrm{O}\right)$ ou l'anhydrite (CaSO4). La mise en solution de ces sels minéraux par les eaux d'infiltration pendant la saison des pluies, favorise leur migration vers les nappes. Ainsi, cette dissolution de minéraux gypseux ou de l'anhydrite serait le deuxième processus d'acquisition des ions $\mathrm{Ca}^{2+}$ dans les eaux souterraines de socle de Natitingou, la première source étant 1'hydrolyse des minéraux silicatés. L'excès de calcium à comparer au contenu de sulfate, observé dans la majorité des échantillons, (Figure 6c) confirme qu'en dehors de l'hydrolyse, il existe d'autres sources de $\mathrm{Ca}^{2+}$ telles que la dissolution du gypse ou de l'anhydrite.

Pour apprécier la part de l'hydrolyse dans l'acquisition des ions, les éléments chimiques majeurs sont mis en relation avec le $\mathrm{pH}$ (Figure 8). En effet, le pH est l'élément indicateur sur les processus chimiques mis en jeu dans la minéralisation. Sa corrélation avec un ion donne une idée précise sur la provenance de cet élément (Abdou Babaye, 2012). On observe que les ions $\mathrm{Ca}^{2+}$ se dégagent des autres par leur corrélation faible avec le $\mathrm{pH}\left(\mathrm{R}^{2}=0,612\right)$, alors que celle-ci est presque nulle avec les ions $\mathrm{Na}^{+}(0,072), \mathrm{K}^{+}(0,191)$ et $\mathrm{Mg}^{2+}(0,2)$ (Tableau 5). La dépendance négligeable de ces trois ions avec le $\mathrm{pH}$ prouve également qu'en dehors de l'hydrolyse, d'autres mécanismes interviennent dans leur mise en solution. 


\begin{tabular}{|c|c|c|c|c|c|c|c|c|c|c|c|c|c|c|c|c|c|c|}
\hline Variables & $\mathrm{pH}$ & $\mathrm{T}^{\circ} \mathrm{C}$ & $\mathrm{CE}$ & $\mathrm{HCO}_{3}$ & $\mathrm{Cl}$ & $\mathrm{NO}_{3}$ & $\mathrm{PO}_{4}$ & $\mathrm{SO}_{4}$ & $\mathrm{Na}$ & $\mathrm{NH}_{4}$ & K & $\mathrm{Mg}$ & $\mathrm{Ca}$ & $\mathrm{NO}_{2}$ & $\mathrm{~F}$ & $\mathrm{Br}$ & TDS & Salinité \\
\hline $\mathrm{pH}$ & 1 & & & & & & & & & & & & & & & & & \\
\hline $\mathrm{T}^{\circ} \mathrm{C}$ & 0,291 & 1 & & & & & & & & & & & & & & & & \\
\hline $\mathrm{CE}$ & 0,487 & 0,152 & 1 & & & & & & & & & & & & & & & \\
\hline $\mathrm{HCO}_{3}$ & 0,725 & 0,427 & 0,446 & 1 & & & & & & & & & & & & & & \\
\hline $\mathrm{Cl}$ & $-0,012$ & $-0,090$ & 0,603 & $-0,060$ & 1 & & & & & & & & & & & & & \\
\hline $\mathrm{NO}_{3}$ & $-0,098$ & $-0,182$ & 0,556 & $-0,152$ & 0,955 & 1 & & & & & & & & & & & & \\
\hline $\mathrm{PO}_{4}$ & 0,078 & 0,140 & 0,284 & 0,209 & 0,182 & 0,150 & 1 & & & & & & & & & & & \\
\hline $\mathrm{SO}_{4}$ & 0,200 & 0,293 & 0,263 & 0,282 & $-0,013$ & $-0,036$ & $-0,025$ & 1 & & & & & & & & & & \\
\hline $\mathrm{Na}$ & 0,072 & 0,104 & 0,731 & 0,057 & 0,749 & 0,686 & 0,197 & 0,173 & 1 & & & & & & & & & \\
\hline $\mathrm{NH}_{4}$ & 0,200 & 0,049 & 0,685 & 0,032 & 0,871 & $\mathbf{0 , 8 0 2}$ & 0,139 & 0,071 & 0,768 & 1 & & & & & & & & \\
\hline K & 0,191 & 0,015 & 0,770 & 0,054 & 0,730 & 0,724 & 0,129 & 0,162 & 0,821 & 0,812 & 1 & & & & & & & \\
\hline $\mathrm{Mg}$ & 0,259 & 0,245 & 0,591 & 0,530 & 0,249 & 0,193 & 0,327 & 0,399 & 0,479 & 0,252 & 0,346 & 1 & & & & & & \\
\hline $\mathrm{Ca}$ & 0,612 & 0,253 & 0,725 & 0,661 & 0,196 & 0,154 & 0,411 & 0,283 & 0,461 & 0,340 & 0,459 & 0,661 & 1 & & & & & \\
\hline $\mathrm{NO}_{2}$ & 0,059 & 0,013 & 0,015 & $-0,023$ & $-0,028$ & $-0,057$ & $-0,039$ & $-0,029$ & 0,083 & 0,067 & $-0,004$ & $-0,056$ & $-0,003$ & 1 & & & & \\
\hline $\mathrm{F}$ & 0,084 & $-0,015$ & 0,317 & 0,235 & 0,325 & 0,277 & 0,364 & 0,278 & 0,278 & 0,277 & 0,202 & 0,524 & 0,280 & $-0,067$ & 1 & & & \\
\hline $\mathrm{Br}$ & 0,111 & 0,011 & $-0,048$ & 0,182 & 0,001 & $-0,068$ & $-0,040$ & $-0,037$ & $-0,047$ & $-0,053$ & $-0,117$ & $-0,058$ & 0,006 & $-0,044$ & $-0,067$ & 1 & & \\
\hline TDS & 0,335 & 0,193 & 0,641 & 0,499 & $-0,068$ & $-0,057$ & 0,224 & 0,398 & 0,338 & $-0,039$ & 0,288 & $\mathbf{0 , 6 3 1}$ & 0,641 & 0,053 & 0,153 & $-0,006$ & 1 & \\
\hline Salinité & 0,388 & 0,137 & 0,932 & 0,290 & 0,670 & 0,605 & 0,284 & 0,278 & 0,743 & 0,776 & 0,794 & 0,464 & 0,592 & $-0,036$ & 0,313 & $-0,069$ & 0,440 & 1 \\
\hline
\end{tabular}

Tableau 5 : Matrice de corrélation entre les variables 

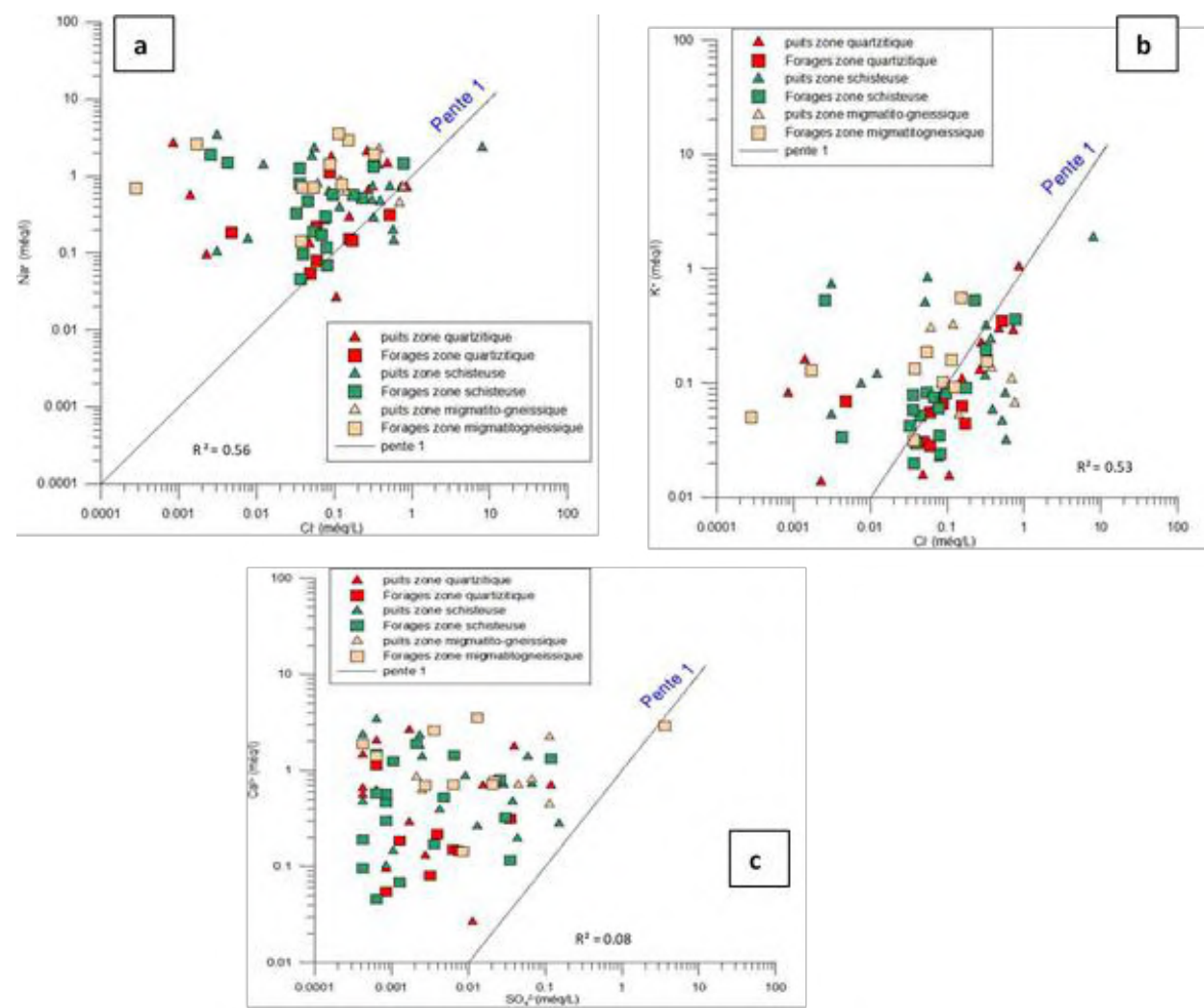

Figure 6 : Relations entre les cations et les bicarbonates

Les phénomènes d'échanges de base qui s'opèrent dans les aquifères de la zone étudiée, sont mis en évidence par la relation $\left[\left(\mathrm{Ca}^{2+}+\mathrm{Mg}^{2+}\right)\right.$ $\left.\left(\mathrm{HCO}_{3}{ }^{-}+\mathrm{SO}_{4}{ }^{2-}\right)\right]$ en fonction de $\left[\mathrm{Na}^{+}+\mathrm{K}^{+}-\mathrm{Cl}^{-}\right]$représentée sur la figure 7 (Garcia et al., 2001). Ces échanges se traduisent par la relation de pente -1 tracée par la position des échantillons. L'opération de soustraction réalisée entre les deux paramètres $\left(\left[\mathrm{Ca}^{2+}+\mathrm{Mg}^{2+}\right)-\left(\mathrm{HCO}_{3}{ }^{-}+\mathrm{SO}_{4}{ }^{2-}\right)\right]$ et $\left.\left[\mathrm{Na}^{+}+\mathrm{K}^{+}-\mathrm{Cl}^{-}\right]\right)$, permet de se débarrasser des ions issus éventuellement d'autres réactions de dissolution des minéraux carbonatés et évaporitiques. En cas d'absence de ces réactions d'échanges de bases, tous les points représentant les échantillons doivent se placer près du point origine selon McLean et al., (2000) comme c'est le cas des eaux des zones quartzitique, et de certains échantillons des zones migmatito-gneissique et schisteuse. On observe le déficit en calcium et l'excès en sodium caractérisant quelques points d'eau prélevés des nappes de la zone schisteuse et de la zone migmatito-gneissique qui peut être en partie attribué aux mécanismes d'échange ionique, par lesquels les ions $\mathrm{Ca}^{2+}$ sont absorbés à la surface des minéraux argileux contre la libération des ions $\mathrm{Na}^{+}$ (Figure 7). Ce phénomène pourrait éventuellement expliquer l'excès des ions $\mathrm{Na}^{+}$par rapport aux ions $\mathrm{Cl}^{-}$(Figure 6a). 


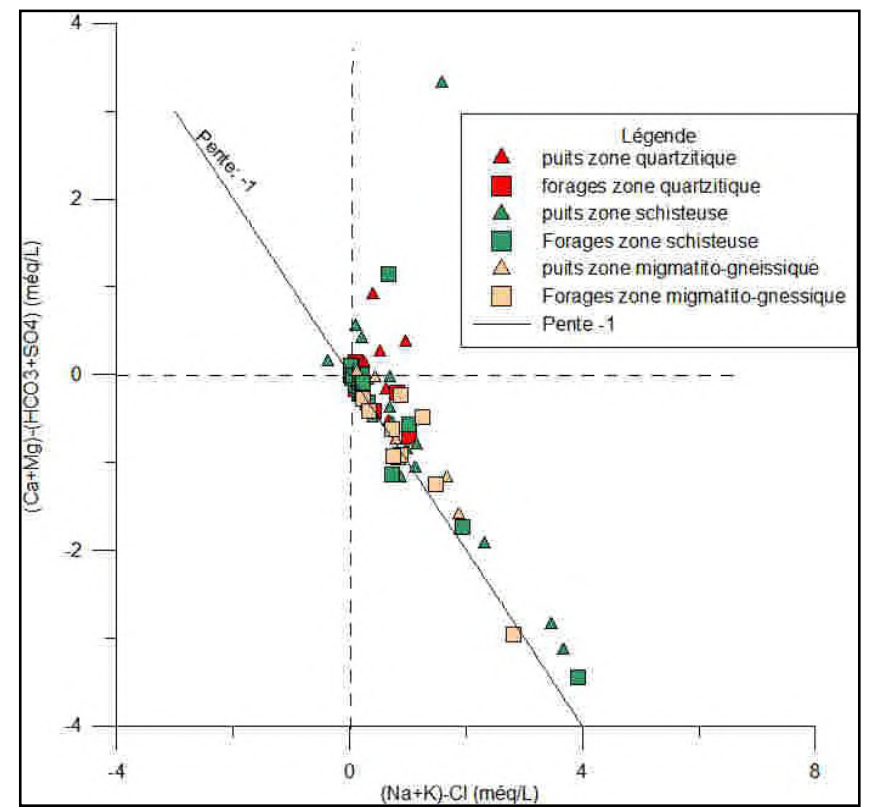

Figure 7 : Relation ionique montrant les échanges cationiques dans les eaux étudiées

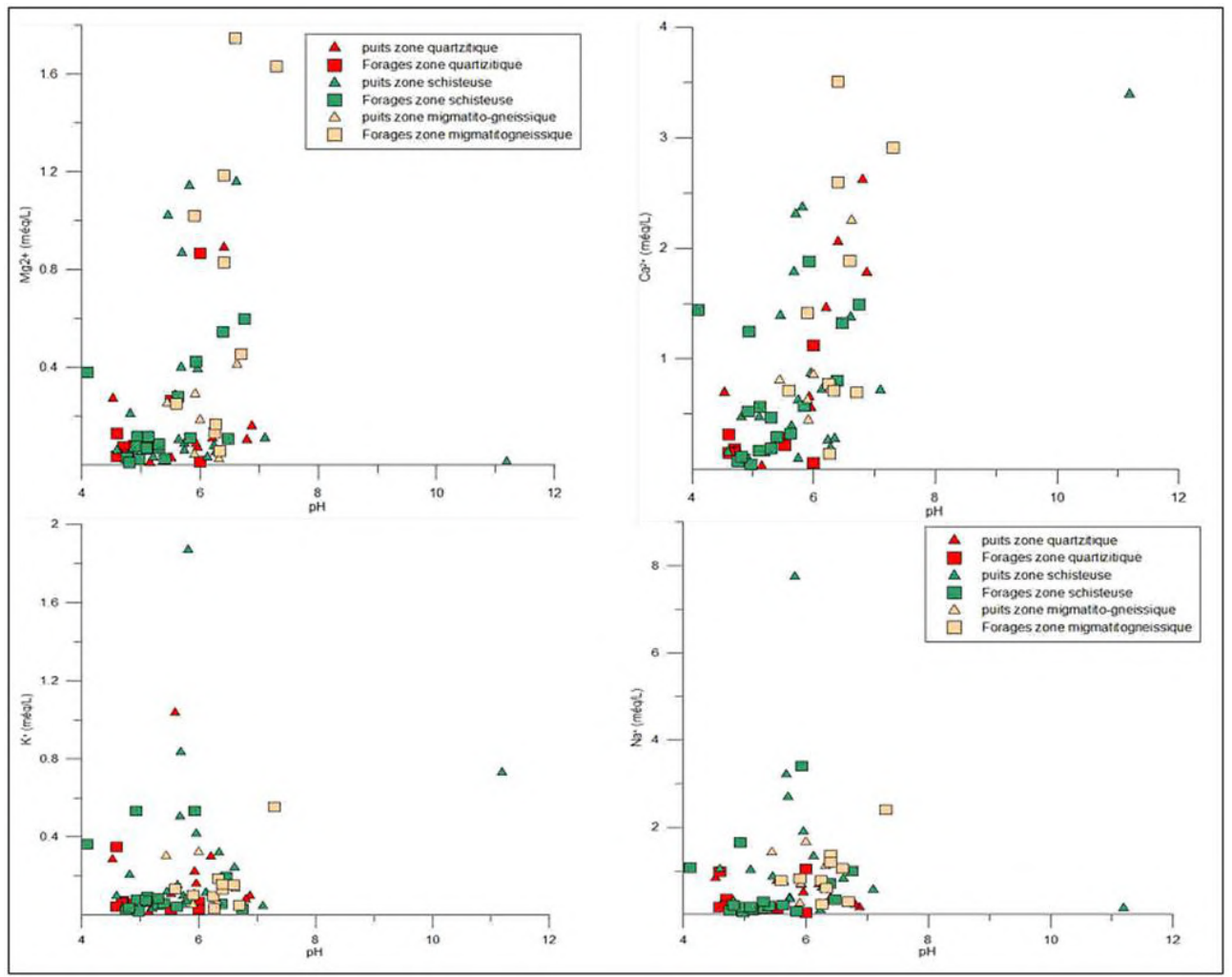

Figure 8 : Relations entre les cations et le $\mathrm{pH}$ 


\section{3- Analyses statistiques}

\section{Matrice de corrélation de Pearson}

La liaison existant entre toutes les variables prises deux à deux et les coefficients de corrélation entre ces différentes variables sont donnés par la matrice de corrélation (Tableau 5). Ce tableau qui présente les différentes corrélations entre les variables peut aider à la compréhension des différents processus intervenant dans la minéralisation des eaux étudiées. Il met en évidence une importante corrélation $\mathrm{NO}_{3}{ }^{-}-\mathrm{Cl}^{-}(0,95)$, salinité-CE $(0,93)$, $\mathrm{NH}_{4}{ }^{+}-\mathrm{Cl}^{-}(0,87), \mathrm{NO}_{3}{ }^{-}-\mathrm{NH}_{4}{ }^{+}(0,80), \mathrm{Na}^{+}-\mathrm{K}^{+}\left(0,82\right.$ et $\mathrm{NH}_{4}{ }^{+}-\mathrm{K}^{+}(0,81)$. Il existe également, mais à un degré moindre, une bonne corrélation $\mathrm{pH}-\mathrm{HCO}_{3}{ }^{-}(0,70)$, $\mathrm{K}-\mathrm{CE}(0,77), \mathrm{Na}^{+}-\mathrm{Cl}^{-}(0,74), \mathrm{K}^{+}-\mathrm{Cl}^{-}(0,73), \mathrm{K}^{+}-\mathrm{NO}_{3}{ }^{-}(0,72), \mathrm{Na}^{+}-\mathrm{NH}_{4}{ }^{+}(0,76)$, salinité- $\mathrm{Na}^{+}(0,74)$, salinité- $\mathrm{NH}_{4}{ }^{+}(0,77)$ et salinité- $\mathrm{K}^{+}$. Ces fortes corrélations observées entre les variables mettent en évidence la similarité des phénomènes à l'origine de la mise en circulation de ces ions dans les eaux souterraines de la zone d'étude. Ces différentes corrélations traduisent l'influence de chaque paramètre dans la minéralisation des eaux de cette commune.

\section{Analyse en Composante Principale}

La variance exprimée 55,62\% par le plan factoriel F1-F2 (Tableau 2) n'est pas très significative pour observer le comportement global des échantillons. Pour cette raison, nous avons aussi considéré les plans factoriels F3-F4 et F4-F5 qui expriment respectivement $14,30 \%$ et $12,53 \%$ de la variance, soit au total 26,83\%, afin de mettre en évidence les autres tendances des échantillons et avoir des projections de bonne qualité (>70\%). Les cinq premiers axes factoriels (F1, F2, F3, F4, F5) expriment 83,45\% (>75\%) de la variance totale. Ce pourcentage est significatif pour faire la classification et étudier les tendances et corrélations qui peuvent exister entre les paramètres et en déduire les mécanismes d'acquisition de la minéralisation dans le milieu.

La projection des variables sur les plans factoriels F1xF2 (Figure 9) montre que le facteur F1 est le plus important. Il contrôle à lui seul, 37,6\% de l'inertie du nuage des points représentatifs des échantillons. Ce facteur regroupe les variables telles que la conductivité électrique $(\mathrm{CE}), \mathrm{Cl}^{-}, \mathrm{Na}^{+}$, $\mathrm{NH}_{4}{ }^{+}, \mathrm{Ca}^{2+}, \mathrm{K}^{+}$, salinité et le $\mathrm{NO}_{3}{ }^{-}$(Tableau 3). Le regroupement de ces variables autour de l'axe F1 montre que cet axe exprime la principale source de minéralisation de l'eau de l'aquifère. On constate par analyse de la principale source de minéralisation deux sous-groupes qui révèlent d'une part l'affinité des ions $\mathrm{Cl}^{-}, \mathrm{NO}_{3}{ }^{-}, \mathrm{NH}_{4}{ }^{+}$et d'autre part celle des ions $\mathrm{Na}^{+}, \mathrm{K}^{+}$. Le premier sous-groupe manifeste l'influence des activités anthropiques dans l'acquisition de la minéralisation des eaux se traduisant surtout par la présence de 1'ion $\mathrm{NO}_{3}{ }^{-}$(Abdou Babaye, 2012 ; Alassane et al., 2015 ; Sambiénou et al., $2018 b)$. Le second sous-groupe provient très souvent de l'hydrolyse des minéraux silicatés surtout en régions de socle. Quant à l'ion $\mathrm{Ca}^{2+}$ exprimé par 
le facteur F1 dont on ne remarque pas l'affinité avec les autres ions $\mathrm{Na}^{+}$et $\mathrm{K}^{+}$, il est aussi généralement fourni par l'hydrolyse des minéraux des formations encaissantes. Cependant, plusieurs auteurs Faillat \& Drogue (1993), Biemi (1992), Yaméogo (2008), Adiaffi (2008), Abdou Babaye (2012), ont démontré qu'une partie non négligeable de $\mathrm{Ca}^{2+}, \mathrm{Na}^{+}$et $\mathrm{K}^{+}$pourrait avoir une origine superficielle (pollution, sols, pluie...) indépendante de l'hydrolyse des minéraux, mais très rarement pour l'ion $\mathrm{Ca}^{2+}$.
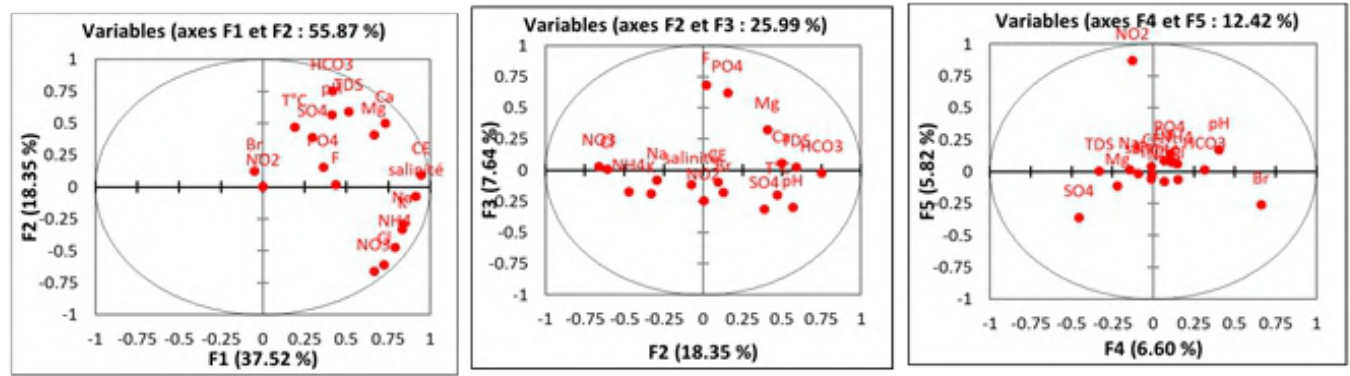

Figure 9 : Analyse dans l'espace des variables des plans factoriels F1-F2 ; F2-F3 et F4-F5.

Le facteur F2 explique environ $18 \%$ de l'inertie totale des points et regroupe les variables $\mathrm{HCO}_{3}, \mathrm{TDS}, \mathrm{pH}, \mathrm{SO}_{4}{ }^{2-}, \mathrm{Mg}^{2+}, \mathrm{T}^{\circ} \mathrm{C}, \mathrm{Ca}^{2+}$ (Figure 9). Ce regroupement met en évidence une minéralisation de l'eau par le phénomène de dissolution de la roche. Selon Abdou Babaye (2012), la corrélation de l'ion $\mathrm{HCO}_{3}{ }^{-}$avec le $\mathrm{pH}(\mathrm{r}=0,72)$, montre que cet ion provient en majorité de l'hydrolyse des minéraux des formations de l'aquifère mais aussi de la diffusion et dissolution du $\mathrm{CO}_{2}$ atmosphérique et du sol. L'ion $\mathrm{SO}_{4}{ }^{2-}$ exprimé par cet axe, pourrait avoir pour origine probable l'oxydation de la pyrite (conditions oxydantes en saison des pluies) (Matini et al., 2009), minéral présent dans les formations géologiques de la région. Leur passage dans l'eau se serait fait suivant la réaction (2) :

$$
\mathrm{FeS}_{2}+15 / 4 \mathrm{O}_{2}+7 / 2 \mathrm{H}_{2} \mathrm{O} \longleftarrow \mathrm{Fe}(\mathrm{OH})_{3}+4 \mathrm{H}^{+}+2 \mathrm{SO}_{4}{ }^{2^{-}} \text {(2) }
$$

$\mathrm{Si}$ les sulfates $\left(\mathrm{SO}_{4}{ }^{2-}\right)$ peuvent provenir des roches (de la zone quartzique et schisteuse), notamment des sulfures, il est aussi vraisemblable que ces anions, en plus des nitrates $\left(\mathrm{NO}_{3}{ }^{-}\right)$, et une partie des cations, pourraient avoir aussi leur origine dans les eaux de pluie et du lessivage des sols par celles-ci. En effet, plusieurs auteurs, Sarazin et al., (1976), Bourrié (1978), Lelong et al., (1989) et Faillat \& Drogue (1993), ont montré que la totalité des anions d'acides forts $\left(\mathrm{Cl}^{-}, \mathrm{SO}_{4}{ }^{2-}, \mathrm{NO}_{3}{ }^{-}\right)$et plus de la moitié des cations $\left(\mathrm{Ca}^{2+}, \mathrm{Mg}^{2+}, \mathrm{Na}^{+}\right)$, pouvaient, en zone de socle, être apportées par les précipitations dans certains cas. De tels bilans hydrochimiques ne peuvent être faits dans cette étude faute d'analyses des eaux de pluies.

Le facteur F3 explique 7,6\% de la variance totale et contient les variables fluorure et orthophosphate. Ce regroupement de variables met également en évidence une minéralisation de l'eau par dissolution des 
minéraux. Selon les auteurs Ravindra \& Garg (2007) et Matini et al., (2009), le fluor dans l'eau provient principalement de la dissolution des minéraux naturels présents dans les roches et les sols avec lesquels l'eau réagit. La fluorine $\left(\mathrm{CaF}_{2}\right)$, la cryolithe $\left(\mathrm{Na}_{3} \mathrm{AlF}_{6}\right)$, le fluoapatite $\left(\mathrm{Ca}_{5} \mathrm{~F}\left(\mathrm{PO}_{4}\right)\right.$ et les micas sont les principaux minéraux qui contiennent l'ion $\mathrm{F}^{-}$(Matini et al., 2009). Les ions fluorures dans les eaux analysées pourraient provenir de la dissolution de ces minéraux car l'ion $\mathrm{F}^{-}$a un rayon ionique identique à celui de l'ion $\mathrm{OH}^{-}$et peut donc se substituer à lui dans ces minéraux (Jordana \& Batista, 2004 ; Matini et al., 2009). Le quatrième facteur F4, quant à lui, contient l'élément $\mathrm{Br}$ et explique $6,60 \%$ de la variance totale. Cet ion, pourrait être également issu de la dissolution des minéraux de roches. Enfin, le facteur F5, contient les ions nitrites $\mathrm{NO}_{2}{ }^{-}$et explique 5,8\% de variance totale. Cet élément tout comme les nitrates pourrait avoir une origine exogène.

La représentation graphique dans l'espace factoriel des individus (Fig. 10) et le tableau 4 rendent compte de la répartition des points d'eau en fonction des différents facteurs F1, F2, F3, F4 et F5. L'analyse de la projection des individus sur les plans F1-F2 et F2-F3, montre trois principaux regroupements des échantillons d'eau concernant leur teneur en des différentes variables étudiées. Le premier regroupement concerne tous les individus dont les teneurs des variables $\mathrm{T}^{\circ} \mathrm{C}, \mathrm{NO}_{3}, \mathrm{TDS}, \mathrm{Cl}, \mathrm{Na}, \mathrm{pH}, \mathrm{HCO}_{3}$, $\mathrm{NH}_{4}, \mathrm{Mg}, \mathrm{Ca}, \mathrm{F}$ et salinité est en dessous de la teneur moyenne. Ce groupe concerne $93,15 \%$ des individus étudiés à l'exception de F33, F35, F3, P5 et P19.

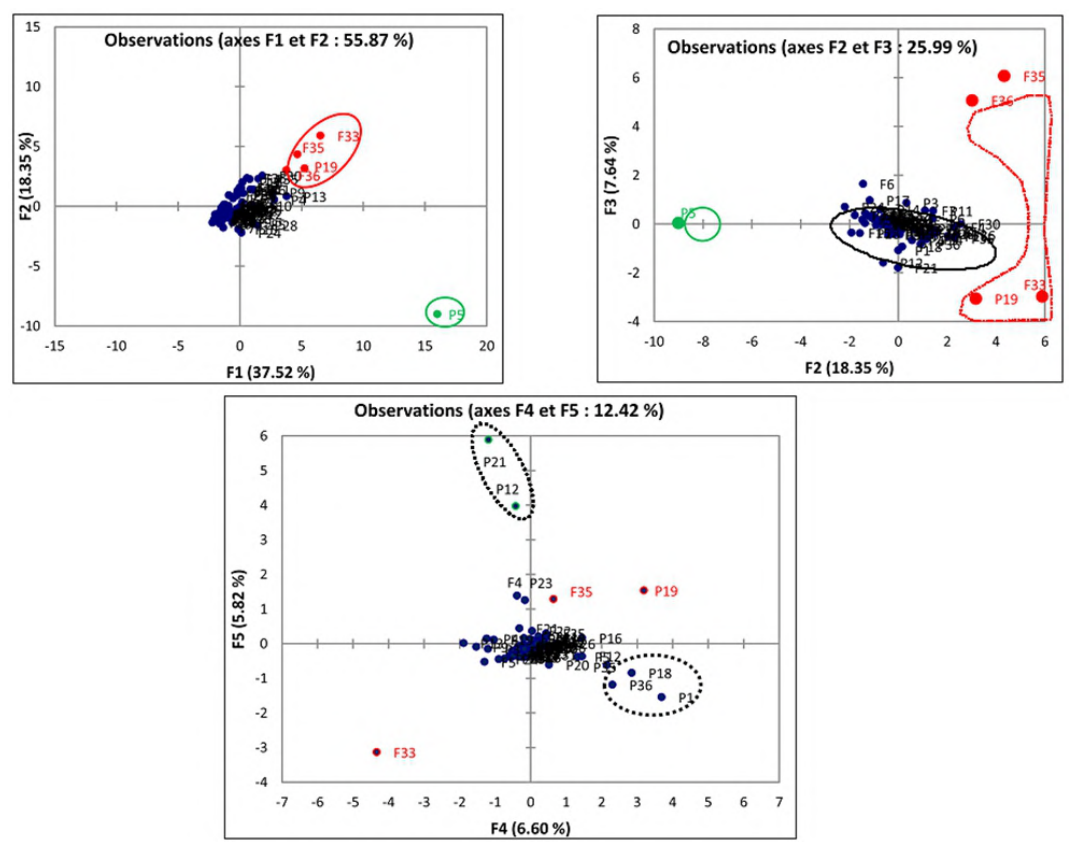

Figure 10 : Analyse dans l'espace des individus des plans factoriels F1-F2 ; F2-F3 et F4-F5 
Le deuxième regroupement qui concerne $6,84 \%$ des individus est caractérisé les échantillons d'eau dont les teneurs des variables $\mathrm{HCO}_{3}, \mathrm{~F}, \mathrm{pH}, \mathrm{SO} 4, \mathrm{PO}_{4}$, $\mathrm{CE}$, salinité, $\mathrm{TDS}, \mathrm{T}^{\circ} \mathrm{C}$ est au-dessus de la teneur moyenne, à l'exception de l'individu P5. Le troisième regroupement qui concerne les individus dont les teneurs en $\mathrm{Cl}$, NO3, NH4, Na, K, salinité, CE et Mg sont largement au-dessus de la moyenne. La projection des individus sur le plan F4-F5 révèle deux autres sous-groupes. Il s'agit du groupe des échantillons P1, P18 et P36 caractérisés par un taux moyen en ion bromure et du groupe des échantillons P12 et P21 se particularisent par un taux élevé en ions nitrites $\mathrm{NO}_{2}$ -

\section{4- Classification Hiérarchique Ascendante (CHA)}

Le dendrogramme (Fig. 11) issu de la Classification Hiérarchique Ascendante (CHA) a mis en évidence quatre principaux regroupements des points d'eau échantillonnés. La première famille qui prend en compte $68 \%$ des échantillons d'eau analysés, est caractérisée par les conductivités électriques inférieures à $500 \mu \mathrm{S} / \mathrm{cm}$. Du point de vue de leur situation géographique, les points d'eau de cette catégorie sont répartis dans les trois zones hydrogéologiques. Ce sont les eaux faiblement minéralisées en majorité. Mis à part les échantillons FO1, FO5, FO7, F16, F18, F19, F20, F21, F36, P5, P14, P15, P22, P23, P26, P28, P33 et P36 qui sont chlorurés, les 32 points d'eau restants de cette classe sont bicarbonatés, soit calciques ou sodiques. Le second regroupement constitué de $22 \%$ des échantillons comprend les eaux dont la conductivité électrique est comprise entre 500 et $1000 \mu \mathrm{S} / \mathrm{cm}$. Cette classe regroupe les eaux à minéralisation importante qui sont également bicarbonatées soit calciques, soit sodiques. La troisième famille qui regroupe environ $7 \%$ des échantillons concerne les eaux à conductivités électriques comprises entre $1000 \mu \mathrm{S} / \mathrm{cm}$ et $2000 \mu \mathrm{S} / \mathrm{cm}(1000<\mathrm{CE}<2000 \mu \mathrm{S} / \mathrm{cm})$. Enfin, le quatrième regroupement d'environ $3 \%$ des échantillons d'eau analysés est caractérisé par les eaux dont la conductivité électrique est supérieure à 2000 $\mu \mathrm{S} / \mathrm{cm}(\mathrm{CE}>2000 \mu \mathrm{S} / \mathrm{cm})$. Les deux dernières classes sont donc caractérisées par les eaux à très forte minéralisation. 


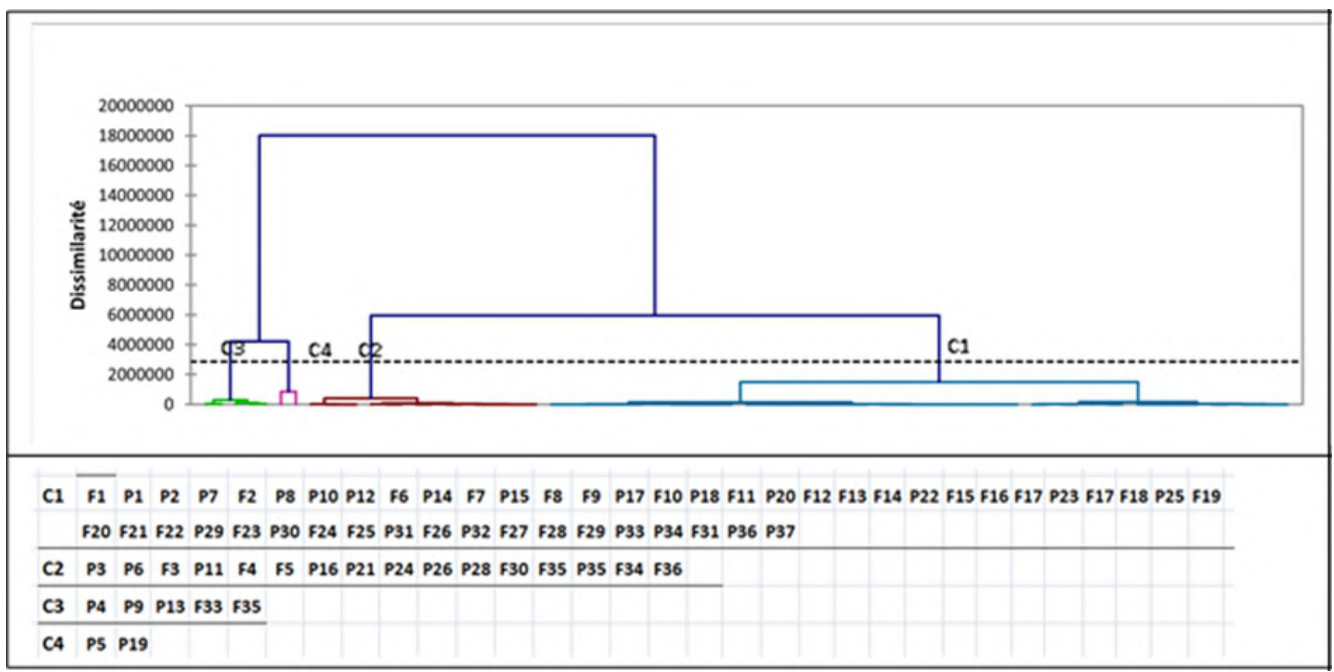

Figure 11 : Dendrogramme de la Classification Hiérarchique Ascendante (CHA) des échantillons d'eau prélevés dans la zone d'étude

Au total, le processus de minéralisation de l'eau est très complexe dans la zone d'étude, du fait d'une part de son contexte géologique et hydrogéologique, à l'origine de la nature discontinue de l'aquifère, d'autre part de la variation spatio-temporelle de la recharge. L'acquisition naturelle des ions du fait de l'altération des roches encaissantes est fortement influencée par un apport exogène d'une partie des cations de l'eau et des ions $\mathrm{Cl}^{-}, \mathrm{NO}_{3}$, $\mathrm{NO}_{2}{ }^{-}, \mathrm{NH}_{4}{ }^{+}$qui proviennent des eaux de pluie et du lessivage des sols par celles-ci comme l'indique les résultats de l'analyse multivariée.

\section{5- Origine anthropique des nitrates : pollution des eaux}

Le tableau 5, montre une très mauvaise corrélation $(r=-0,068)$ entre les ions chlorures et les TDS. Cette observation traduirait l'existence d'origine(s) autre(s) que celles d'une minéralisation naturelle en ces ions. Cependant, la corrélation très significative $(\mathrm{r}=0,95)$, notée entre les ions $\mathrm{NO}_{3}{ }^{-}$ et $\mathrm{Cl}^{-}$prouve que ces deux ions proviennent d'une même origine. De l'analyse statistique, l'affinité des ions $\mathrm{Cl}^{-}, \mathrm{NO}_{3}{ }^{-}$et $\mathrm{NH}_{4}{ }^{+}$observée en projetant les variables sur l'axe F1, manifeste l'influence des activités anthropiques dans l'acquisition de la minéralisation des eaux se traduisant surtout par la présence de l'ion $\mathrm{NO}_{3}$. Le tableau 1, montre que sept échantillons P5 (437,72 mg/L), P24 (133 mg/L), P28n (97,78 mg/L), F5 (96,28 mg/L), P7(70,37 mg/L), P14 $(50,78 \mathrm{mg} / \mathrm{L})$ et P15 $(54,7 \mathrm{mg} / \mathrm{L})$ désignant respectivement les localités de Djindjirébéri, Kotopounga, Banguirétamou, Winkè, Boriyouré, Kantaborifa et Ouroubouga présentent des concentrations en $\mathrm{NO}_{3}{ }^{-}$supérieures à la valeur guide de l'O.M.S qui est de $50 \mathrm{mg} / \mathrm{L}$ pour l'eau de boisson (WHO, 2008). On remarque que les échantillons à forte teneur en $\mathrm{NO}_{3}{ }^{-}$sont majoritairement ceux 
des puits traditionnels qui captent la nappe superficielle d'altérites très accessible par les apports exogènes. De plus, les localités concernées ici, sont pour la plupart des quartiers de la ville de Natitingou ou le siège d'arrondissement où l'influence humaine très marquée participe à la pollution de la nappe.

\section{Conclusion}

L'étude réalisée sur les eaux souterraines de la commune de Natitingou a permis de connaître leurs différentes caractéristiques. Elles sont acides à neutre avec une minéralisation importante dont la moyenne est de 465,64 $\mu \mathrm{S} / \mathrm{cm}$. La classification hydrochimique des eaux a montré une dominance du faciès bicarbonaté calcique suivi du faciès bicarbonaté sodique. Le faciès chloruré, représenté à $25 \%$, traduit l'apport anthropique dans l'acquisition de la minéralisation. Cela se confirme également par l'interprétation des ACP qui précise que les eaux des localités de Djindjirébéri, Kotopounga, Banguirétamou, Winkè, Boriyouré, Kantaborifa et Ouroubouga, siège d'arrondissement où l'influence humaine est très marquée, sont polluées.

\section{Remerciements}

Nous remercions le Laboratoire d'Hydrologie Appliquée pour son appui financier lors des travaux de terrain et d'analyses chimiques. Nos vifs remerciements vont également à l'endroit des lecteurs anonymes pour leurs précieuses contributions.

\section{References:}

1. Abdou Babaye M.S. (2012). Evaluation des ressources en eau dans le bassin de Dargol (Liptako-Niger). Thèse de Doctorat, Université de Liège \& Université Abdou Moumouni, 265p.

2. Abid K. (2010). Identification et caractérisation hydrogéologique et géochimique de la nappe du Continental Turonien dans le Sud tunisien et sa relation avec les aquifères adjacents. Thèse de Doctorat, Ecole Nationale d'Ingénieurs de Sfax, Tunisie, 192p.

3. Adiaffi B. (2008). Apport de la géochimie isotopique, de l'hydrochimie et de la télédétection à la connaissance des aquifères de la zone de contact "socle-bassin sédimentaire" du Sud Est de la Côte d'Ivoire. Thèse de doctorat, Univ. Paris Sud, 230p.

4. Affaton P. (1987). Le Bassin des Volta (Afrique de l'Ouest): une marge passive, d'âge protérozoïque supérieur, tectonisée au Panafricain (600 $\pm 50 \mathrm{Ma})$. Thèse de doctorat, Editions de l'ORSTOM, Collection Etudes et Thèses, Paris, 496 p.

5. Ahissou S.R. \& Goudalo N. (2004). Potentialités hydrogéologiques en relation avec les ensembles pétrographiques et la tectonique du 
département de l'Atacora. Mémoire de maîtrise en Géologie Fondamentale et Appliquée, Université d'Abomey Calavi, 55p.

6. Alassane A., Trabelsi R., Dovonon L.F., Odeloui D.J., Boukari M., Zouari K., Mama D. (2015). Chemical Evolution of the Continental Terminal Shallow Aquifer in the South of Coastal Sedimentary Basin of Benin (West-Africa) Using Multivariate Factor Analysis. Journal of Water Resource and Protection, 7, 496-515.

7. Biémi J. (1992). Contribution à l'étude géologique, hydrogéologique et par télédétection des bassins versants subsahéliens du socle Précambrien d'Afrique de l'Ouest: hydrostructurale, hydrodynamique, hydrochimie et isotopie des aquifères discontinus de sillons et aires granitiques de la haute Marahoué (Côte d'Ivoire). Thèse de doctorat, Univ. Natio. Côte d'Ivoire, 493p.

8. Bonhomme M., (1962). Contribution à l'étude géochronologique de la plateforme de l'Ouest africain. Ann. Fac. Sc., Université de ClermontFernand, Géol. Minéral. N5, France.

9. Bourrié G. (1978). Acquisition de la composition chimique des eaux en climat tempéré. Application aux granites des Vosges et de la Margeride. Persée-Portail des revues scientifiques en SHS, 52, 1.

10. Eblin S.G., Soro G.M., Sombo A.P., Aka N., Kambiré O., Soro N. (2014). Hydrochimie des eaux souterraines de la région d'Adiaké (sudest côtier de la côte d'ivoire). Larhyss Journal, ISSN 1112-3680, n¹7, pp. 193-214.

11. Faillat J.P. \& DROGUE C. (1993). Différenciation hydrochimique de nappes superposées d'altérites et de fissures en socle granitique. Hydrological sciences journal, 38, 3, pp. 215-229.

12. Freeze R.A., Cherry J.A. (1979). Groundwater. Prentice-Hall, Inc, Englewood Cliffs, NJ, 604p.

13. García G.M., Hidalgo M.D.V., Blesa M.A. (2001). Geochemistry of groundwater in the alluvial plain of Tucuman province, Argentina. Hydrogeology Journal, 9(6), pp. 597-610.

14. Géohydraulique (1985). Hydraulique villageoise dans les provinces de l'Atacora, du Mono et de l'Ouémé, rapport final, Direction de l'Hydraulique, Cotonou, Bénin, 236 p.

15. Jordana S. \& Batista E. (2004). Natural groundwater quality and health. Geologica Acta: an international earth science journal, 2, 2, pp. 175-188.

16. Lelong F., Durand P., Vannier C., Guillet B., Rouiller J. (1989). Importance de la rétention des ions sulfates dans des sols granitiques acides en région de moyenne montagne (Mont Lozère): relation générale avec le pouvoir tampon des sols. Comptes rendus de 
l'Académie des sciences. Série 2, Sciences de l'univers, Sciences de la Terre, 309(19), 1991-1996.

17. Matini L., Moutou J.M., Kongo-Mantono M.S. (2009). Evaluation hydro-chimique des eaux souterraines en milieu urbain au Sud-Ouest de Brazzaville, Congo. Afrique Science: Revue Internationale des Sciences et Technologie, 5, 1 .

18. McLean W., Jankowski J., Lavitt N. (2000). Groundwater quality and sustainability in an alluvial aquifer, Australia. Groundwater, past achievements and future challenges. A Balkema, Rotterdam, 567-573.

19. Pougnet R. (1957). Le précambrien du Dahomey Bulletin de la Direction Fédérale des Mines et de la Géologie de l'Afrique Occidentale Française.

20. Ravindra K., Garg V.K. (2007). Hydro-chemical survey of groundwater of Hisar city and assessment of defluoridation methods used in India. Environmental monitoring and assessment, 132(1-3), 33-43.

21. Sambiénou W.G., Orou Pete Allou G.S., Alassane A., Mama D., Boukari M. (2018). Caractérisation hydrogéochimique des eaux souterraines du bassin versant de Pendjari (nord-ouest du Bénin), Revue Internationale des Sciences Appliquées, ISSN-1840-8869, Vol $1, \mathrm{n}^{\circ} 02, \mathrm{pp} 18-30$.

22. Sambiénou W.G. (2019). Contribution à la compréhension du fonctionnement et à l'évaluation des potentialités en eau du système aquifère du bassin versant de la Pendjari (Nord-Ouest du Bénin) : Apports des outils piézométriques, hydrogéochimiques, isotopiques et de la télédétection. Thèse de Doctorat, Université d'Abomey-Calavi, CIPMA, Bénin, 202p.

23. Sarazin G., Fouillac C., Michard G. (1976). Etude de l'acquisition d'éléments dissous par les eaux de lessivage des roches granitiques sous climat tempéré. Geochimica et Cosmochimica Acta, 40, 12, pp.1481-1486.

24. SOGREAH-SCET-Tunisie. (1997). Etude de la stratégie nationale de gestion des ressources en eau du Bénin ; Rapports, R1 à R7. Cotonou, Bénin.

25. WHO. (2008). Guidelines for Drinking water Quality. Third Edition Incorporating the First and Second Addenda. In: Recommendations, vol. 1, Geneva.

26. Yaméogo O.S. (2008). Ressources en eau souterraine du centre urbain de Ouagadougou au Burkina Faso, qualité et vulnérabilité. Thèse de doctorat, Univ. Avignon et pays de Vaucluse, Univ. Ouagadougou, $245 p$. 